\title{
OPTIMIZATION OF BEAM-SHAPING ASSEMBLIES FOR BNCS USING THE HIGH-ENERGY NEUTRON SOURCES D-D AND D-T ${ }^{1}$
}

\author{
J.M. Verbeke ${ }^{a, b}$, A. Chen ${ }^{a, b}$, J. Vujic $^{a}$ and K.N. Leung ${ }^{b}$ \\ ${ }^{a}$ Nuclear Engineering Department, University of California, Berkeley \\ ${ }^{b}$ Lawrence Berkeley National Laboratory \\ Berkeley, CA 94720, USA
}

Author to whom correspondence should be sent:

J.M. Verbeke

1641 Belleville Way, Apt P

Sunnyvale, CA 94087

email: jerome.verbeke@eng.sun.com

phone: (408) 2455692

Number of pages: 49

Number of tables: 2

Number of figures: 18

\footnotetext{
${ }^{1}$ This work is supported by the U.S. Department of Energy under contract DE-AC03-76SF00098
} 


\begin{abstract}
Boron neutron capture synovectomy is a novel approach for the treatment of rheumatoid arthritis. The goal of the treatment is the ablation of diseased synovial membranes in articulating joints. The treatment of knee joints is the focus of this work. A method was developed, as discussed previously, to predict the dose distribution in a knee joint from any neutron and photon beam spectra incident on the knee. This method is validated and used to design moderators for the deuterium-deuterium (D-D) and deuterium-tritium (D-T) neutron sources. Treatment times $>2 \mathrm{~h}$ were obtained with the D-D reaction. They could potentially be reduced if the ${ }^{10} \mathrm{~B}$ concentration in the synovium was increased. For D-T neutrons, high therapeutic ratios and treatment times $<5$ min were obtained for neutron yields of $10^{14} \mathrm{~s}^{-1}$. This treatment time makes the D-T reaction attractive for boron neutron capture synovectomy.
\end{abstract}




\section{INTRODUCTION}

Rheumatoid arthritis is a disease characterized by the inflammation of the synovial membrane or synovium, a thin tissue layer that overlays articulating joints (such as knee and finger joints) and provides lubrication for the articulation. It results in swollen, inflamed, and painful joints. Different treatments are currently used for diseased knee joints, the subject of this paper. The most common ones are the administration of drugs to reduce synovial inflammation and the

excision of the inflamed synovium via invasive surgery. While some patients are unresponsive to the former treatment, the latter presents disadvantages, such as potential infection, hemorrhage, anesthesia, and long rehabilitation periods. Radiation synovectomy is an alternative treatment that uses beta-emitting radionuclides. It presents several advantages, such as success rates comparable to surgery, local anestesia, no rehabilitation time, lower cost, and a less time-consuming procedure. However, irradiation of healthy tissues by diffusion of beta-emitters away from the joint is a major concern $[1,2]$.

Boron neutron capture synovectomy (BNCS) $[3,4,5,6]$ is currently being investigated as an alternative treatment for rheumatoid arthritis. It employs the ${ }^{10} \mathrm{~B}(n, \alpha){ }^{7} \mathrm{Li}$ reaction, which has a large cross section for thermal neutrons. A boronated compound such as $\mathrm{K}_{2} \mathrm{~B}_{12} \mathrm{H}_{12}$ is injected into the synovial membrane of the diseased knee, which is then exposed to a low-energy neutron beam. Boron-10 atoms undergo fission reactions and release high-energy, high-linearenergy-transfer alpha particles and lithium nuclei, which deposit their energy locally (typically 2.3 to $2.8 \mathrm{MeV}$ within 4 to $9 \mu \mathrm{m}$ ) damaging or killing cells along their paths. The dose to the synovium is significantly enhanced by the higher concentration of ${ }^{10} \mathrm{~B}$ in the synovium. BNCS offers the same advantages as therapies based on beta-emitting radionuclides by being noninvasive, and additionally by permitting a better control on the irradiation of healthy tissues, since cell killing is triggered by the neutron beam and stops after irradiation.

In this paper, the candidate neutron sources deuterium-deuterium (D-D) and deuterium- 
tritium (D-T) are being investigated for BNCS. The advantage of these nuclear reactions is the low interaction energy required for them to occur, resulting in high neutron yields even for low-energy particle accelerators of 100 to $200 \mathrm{keV}$. However, the neutrons produced by these reactions have energies of 2.45 and $14.1 \mathrm{MeV}$, respectively, which present challenges for their moderation to the thermal energies required for BNCS (Ref. [6]). The design of beam-shaping assemblies (BSAs) for high-energy neutron moderation is the subject of this paper.

\section{FIGURES OF MERIT}

To be efficient, the neutron beam must deliver a dose of 100 Gy-equivalent to the boron-loaded synovium. This dose level corresponds to the dose required to produce a clinical effect on the synovium using beta-emitters. Boronated compounds uptake in the synovium higher than 1000 ppm have been reported [6]. With this localized high boron uptake, high doses to the synovial membrane can be achieved without irradiating excessively neighboring tissues such as skin and bone.

Radiation effects in the skin are nonstochastic and a mild skin reddening is observed at doses of approximately 8 Gy-equivalent (Ref. [7]). Concerning the bone, the probability of cancer induction increases with the radiation dose without dose threshold [3]. Reduction of bone dose is crucial to the success of any BNCS treatment regimen.

Let $\mathrm{D}_{\text {syn }}, \mathrm{D}_{s k}$ and $\mathrm{D}_{\text {bone }}$ be the average absorbed synovium, skin, and bone doses per neutron emitted by the source. Let $\mathrm{N}$ be the number of source neutrons required to reach the 100 Gy-equivalent dose to the synovium; $\mathrm{N}$ is determined using the following equation:

$$
N \cdot D_{\text {syn }}=100 \text { Gy-equivalent }
$$

All the doses are scaled by $\mathrm{N}$ in such a way that Eq. (1) is verified. The synovium, skin, and bone doses are then given by $\mathrm{N} \cdot \mathrm{D}_{s y n}, \mathrm{~N} \cdot \mathrm{D}_{s k}$, and $\mathrm{N} \cdot \mathrm{D}_{\text {bone }}$, respectively. From Eq. (1), one 
can compute the time $\mathrm{T}$ required for treatment using

$$
T=\frac{N}{S}(s)
$$

where $\mathrm{S}$ is the neutron source strength in units per second. For the sake of clarity, all symbols used in the text are included in Table I.

An analysis based on two figures-of-merit has been proposed by Yanch [3] to measure the beam quality. The first one is the ratio of the synovium-absorbed dose to the skin-absorbed dose $\mathrm{D}_{\text {syn }} / \mathrm{D}_{\text {sk }}$. It should be $>12.5$ to satisfy both the 100 Gy-equivalent dose to the synovium and the 8 Gy-equivalent dose limit on the skin. The second is the ratio of the synovium-absorbed dose to the bone-absorbed dose $\mathrm{D}_{\text {syn }} / \mathrm{D}_{\text {bone }}$. This ratio should be maximized to limit potential cancer induction.

Besides maximizing the ratio $\mathrm{D}_{\text {syn }} / \mathrm{D}_{\text {bone }}$ under the constraint $\mathrm{D}_{\text {syn }} / \mathrm{D}_{\text {sk }} \geq 12.5$, an ideal neutron beam must have sufficient intensity for short treatment times, although fractionated radiation schemes could be adopted.

Doses in the knee were computed using two different methods. The first method, referred to as the full-simulation method (or Full Sim in the figures), simulates the particle transport from the neutron source to the knee through the BSA. The second method, referred to as doseresponse database simulation method (or DRD Sim in the figures), was developed in a previous paper [8]. It predicts the dose distribution in the knee, based on the energy distributions of the neutron and photon beams incident on the knee. This method reduces the particle transport simulation time by a factor of 10 relative to the full-simulation method. Both methods are based on the human knee model taken from Ref. [5] and shown in Fig. 1. The synovium layer is located between 1.3 and $1.45 \mathrm{~cm}$ below the surface of the skin. The elemental compositions of tissue and bone are given in Ref. [5]. Boron-10 concentrations of $1000 \mathrm{ppm}$ in the synovium and $1 \mathrm{ppm}$ in all other tissues are assumed. The neutron beam direction is normal to the skin and is $8.7 \mathrm{~cm}$ in diameter. 
For the full-simulation method, neutron and photon transport in the soft tissues was simulated by the Monte-Carlo code MCNP [9]. The neutron and photon fluences computed in the volumes inside the 2.5-cm-diam cylinder intersecting the knee (see Fig. 1) are modified by the fluence-to-kerma conversion factors and the photon mass attenuation coefficients of the International Commission on Radiological Units and Measurments (ICRU) report 46 (Ref. [10]) to compute the neutron doses $\mathrm{D}_{n}$ and photon doses $\mathrm{D}_{\gamma}$. The dosimetric effect of neutrons will depend on the concentrations of ${ }^{10} \mathrm{~B}$ in both synovium and healthy tissues. To estimate the ${ }^{10} \mathrm{~B}(n, \alpha){ }^{7} \mathrm{Li}$ contribution $\mathrm{D}_{B}$ to the dose, the neutron fluence was modified by ${ }^{10} \mathrm{~B}$ fluence-tokerma conversion factors, listed in Caswell et al. [11], and then multiplied by either a factor of $1 \mathrm{ppm}$ (to represent the $1 \mu \mathrm{g} / \mathrm{g}$ of ${ }^{10} \mathrm{~B}$ in healthy tissues) or a factor of $1000 \mathrm{ppm}$ (to represent the $1000 \mu \mathrm{g} / \mathrm{g}$ of ${ }^{10} \mathrm{~B}$ in synovium). Dose-response curves depend on the type of radiation used and on the biological endpoint studied. Different radiations can be contrasted in terms of their relative biological effectiveness (RBE) compared with $\mathrm{X}$ rays. If a dose $\mathrm{D}$ of a given type of radiation produces a specific endpoint, then $\mathrm{RBE}$ is defined as the ratio $\mathrm{RBE}=\mathrm{D}_{X} / \mathrm{D}$, where $\mathrm{D}_{X}$ is the $\mathrm{X}$-ray dose needed under the same conditions to produce the same endpoint. The total absorbed tissue doses are obtained by combining the individual dose components weighted by their RBE factors, using the following equation:

$$
D_{\text {total }}=R B E_{B} \cdot D_{B}+R B E_{n} \cdot D_{n}+R B E_{\gamma} \cdot D_{\gamma} \quad(G y-\text { equivalent per neutron }),
$$

where the following assumptions are made: ${ }^{10} \mathrm{~B}$ reaction products $R B E_{B}$ is 4.0 , neutron reaction $R B E_{n}$ is $3.8, R B E_{\gamma}$ is 1.0 . The $\mathrm{RBE}$ values and ${ }^{10} \mathrm{~B}$ concentrations were taken directly from values used in Ref. [5]. 


\section{DESIGN OF BSAS FOR BNCS FOR THE D-D AND D-T NEUTRON SOURCES}

A previous study [3] showed that the fusion reactions D-D and D-T did not fulfill the requirements for BNCS, i.e., high therapeutic ratios and short treatment times. Based on the experience acquired on moderation of high-energy neutrons in the context of boron neutron capture therapy [12] (BNCT), it was believed that the BSAs used in this study were not optimal. Therefore, the use of D-D and D-T neutrons for BNCS was reinvestigated in light of our work for BNCT.

Previous studies [6] showed that thermal neutron beams were optimal for BNCS treatment. Neutrons from both fusion reactions have thus to be moderated down to thermal energies. The following sections focus on designing BSAs to shape the most suitable neutron beam. Sections III.1 and III.2 describe the neutron source and the BSA; Sec. III.3 concentrates on the designs of the delimiter and the front-reflector; Sec. III.4 explains the methodology used to optimize the BSA; and Secs. III.5 and III.6 focus on the moderation of D-D and D-T neutrons.

\section{III.1 Neutron source characterization}

In our numerical simulations, the neutron source is characterized as follows. Neutrons are emitted isotropically and monoenergetically across a 5-cm-diam flat circular surface. The source is distributed uniformly over the surface of the target, which is composed of a $10-\mu \mathrm{m}$-thick titanium layer on a 1-mm-thick copper substrate water-cooled on the back by 3 -mm-deep water channels machined in a 5-mm-thick stainless steel plate. The spread in the D-D and D-T neutron energies due to deuteron straggling in the target is not considered in this study. The assumption of isotropy has to be discussed in detail. Concerning the D-T reaction, the high $Q$ value for the reaction makes the neutron energy relatively insensitive to the angle of emission for the region of low deuteron energy $(\sim 100 \mathrm{keV})[13]$. The neutron energy varies around $14.1 \mathrm{MeV}$ by only $\pm 7 \%$ over all solid angles for a deuteron beam energy of $200 \mathrm{keV}$. The neutrons are emitted practically 
isotropically in the center-of-mass system below this energy. In the lab system, the differential cross section for the $\mathrm{D}-\mathrm{T}$ reaction varies by only $\pm 7 \%$ for the same $200 \mathrm{keV}$ deuteron beam. Thus, angular isotropy in the lab system is an adequate approximation for deuteron beams of low energy. For D-D, the angular distribution in the center-of-mass system is anisotropic. A better modeling of the source accounting for the angular distribution would be required, but this is beyond the scope of this work.

\section{2 BSA description}

Source neutrons enter a cylindrical BSA with the monoenergetic neutron distribution corresponding to D-D or D-T reactions. They travel through the BSA, which contains several layers of different materials until they reach the other side where the knee is located (see Fig. 2). A thick lithiated polyethylene [14] layer — referred to as the neutron beam delimiter further in the text - separates the BSA from the knee in order to restrict the photon and neutron fluxes to a 8.7 -cm-diam window, which corresponds to the diameter of the knee. The role of this layer is to reduce radiation exposure to the organs other than the knee. The collimator (shown in Fig. 2 as a truncated cone) is located between the moderator and the exit window. It can be made out of the same material as the delimiter or out of any other material such as graphite or lead. Its role is to collimate the neutron beam to the exit window. A front-reflector can be placed between the moderator and the delimiter to increase the neutron flux at the end of the moderator and consequently the neutron current at the exit window.

A generic BSA is used for the neutron beam delimiter and front-reflector optimization. It is shown in Fig. 3. This BSA is composed of a 50-cm-thick, 35-cm-diam $\mathrm{D}_{2} \mathrm{O}$ moderator surrounded by a 10 -cm-thick lead reflector, which is in turn surrounded by a 10-cm-thick lithiated polyethylene layer. The back-reflector is a $10-\mathrm{cm}$-thick lead layer and is followed by a $10-\mathrm{cm}$-thick lithiated polyethylene layer. The delimiter between the BSA and the knee is a single 11-cm-thick 
lithiated polyethylene layer. No front-reflector is used in this generic BSA. The collimator is cylindrical, $8.7 \mathrm{~cm}$ in diameter, and made out of the same material as the delimiter.

\section{III.3 Neutron beam delimiter and front-reflector}

The role of the delimiter (see Fig. 3) is to decrease radiation exposure to the organs other than the knee. It has to attenuate both neutron and photon fluxes coming out of the moderator efficiently. Lithiated polyethylene is commonly used to reduce photon fluxes. The light elements $\mathrm{C}$ and $\mathrm{H}$ thermalize the neutrons, while lithium enriched in its isotope ${ }^{6} \mathrm{Li}$ absorbs the thermalized neutrons.

The role of the front-reflector is to reflect back to the moderator some neutrons that would otherwise be lost in the delimiter. Materials of choice for this purpose are the same as the ones used to reflect neutrons in reactors, i.e., graphite, lead, etc. Front-reflectors are not commonly used in BSAs but appeared to be beneficial to the treatment in this study.

\section{III.3.1 Delimiter and front-reflector thicknesses and materials}

Using the generic BSA shown in Fig. 3 and described in Sec. III.2, along with the D-D neutron source, several simulations are performed using lithiated polyethylene delimiters with no frontreflectors, and using graphite and lead front-reflectors with no delimiters.

Two quantities are used to compare the performances of the delimiters and front-reflectors.

The first quantity measures the decrease in neutron current across the exit window due to the presence of the delimiter (or front-reflector). To define it mathematically, we must first define

$\mathrm{I}_{n}$ across exit window as the neutron current across the exit window (to be distinguished from the neutron current density across the exit window). This first quantity then becomes the ratio

$$
R_{\text {exit window }}=\frac{I_{n \text { across exit window }} \text { with delimiter } / \text { front }- \text { reflector }}{I_{n \text { across exit window }} \text { without delimiter } / \text { front }- \text { reflector }}
$$

i.e., the ratio of the neutron current across the exit window with the delimiter/front-reflector 
to the same current without any delimiter/front-reflector. Note that since the exit window is at the end of the delimiter/front-reflector, its position varies with the delimiter/front-reflector thickness.

The second quantity measures the delimiter (or front-reflector) "attenuation efficiency". It is defined as the ratio

$$
R_{\text {attenuation efficieny }}=\frac{I_{n \text { across exit window }}}{I_{n \text { around exit window }}}
$$

where $\mathrm{I}_{n}$ around exit window is the neutron current in the annular area of inner diameter equal to the exit window diameter $(8.7 \mathrm{~cm})$ and outer diameter equal to the reflector outer diameter $(55 \mathrm{~cm})$. Since the ratio of the areas is $\pi \cdot 4.35^{2} / \pi \cdot\left(27.5^{2}-4.35^{2}\right) \simeq 1 / 39$, the ratio of the neutron current density across the exit window to the one around the exit window can be found by multiplying the neutron attenuation efficiency by 39 .

The first simulations are performed with lithiated polyethylene delimiters of different thicknesses. The results are shown in Figs. $4 \mathrm{a}$ and $4 \mathrm{~b}$. The value $\mathrm{R}_{\text {exit window }}$ decreases as the delimiter thickness increases, while the directionality of the beam increases, see Fig. 5. This means that most of the neutrons hitting the walls of the collimator are eventually absorbed in the delimiter. On the contrary, if graphite or lead is used as a front-reflector, the ratio $\mathrm{R}_{\text {exit window }}$ first increases to reach a maximum of 1.16 for a thickness of $4 \mathrm{~cm}$ and then slowly decreases, while the directionality of the beam slowly but steadily increases. This small increase can be qualitatively explained. The lead and graphite layers reflect neutrons - that would otherwise be absorbed in the lithiated polyethylene - back to the moderator. These neutrons then contribute to the neutron current at the exit window of the collimator. This leads to the conclusion that the neutron current across the exit window can be increased by $\sim 16 \%$ by inserting a layer of either graphite or lead between the moderator and the delimiter. This layer — shown as front-reflector in Fig. 2 - will systematically be added to our BSAs. While lead is advantageous for the attenuation of photons, graphite is preferable when photons are not an 
issue, since the density of graphite makes it mechanically much easier to handle.

Figure $4 \mathrm{~b}$ shows that the neutron attenuation efficiency steadily increases for lithiated polyethylene. Lead and graphite are very poor materials in that regard. A 8-cm-thick layer of lithiated polyethylene will be used in our BSAs to reduce neutron leakage. This will lead to an average neutron current density 60 times lower outside the exit window than across the exit window.

\section{III.3.2 Collimator thickness and angle}

Using a 5-cm-thick graphite front-reflector followed by a 8-cm-thick layer of lithiated polyethylene as a delimiter, we now study the influence of the angle $\alpha$ and the thickness of the collimator (as shown in Fig. 2). The material used for the collimator is graphite. A 2-cm-thick layer of lithiated polyethylene (see Fig. 2) is left between the collimator and the knee to compensate for the lower neutron attenuation of the collimator material. Figure $6 \mathrm{a}$ shows the ratio $\mathrm{R}_{\text {exit window }}$ for different collimator angles and thicknesses. This ratio, which measures the neutron output gain or loss due to the front reflector and delimiter, increases steadily with the collimator angle $\alpha$ until it reaches its maximum at an angle of $40 \mathrm{deg}$, and then starts decreasing. It also increases with the collimator thickness. A thick collimator of angle $40 \mathrm{deg}$ seems to be optimal for neutron collimation. However, Fig. $6 \mathrm{~b}$ shows that the neutron attenuation efficiency decreases steeply with the collimator angle, past a critical angle of $20 \mathrm{deg}$.

Thin collimators have lower attenuation efficiencies for small angles, while they have higher ones for large angles. This can qualitatively be explained by the fact that some neutrons are reflected off the walls of the graphite collimator and directed toward the exit window,

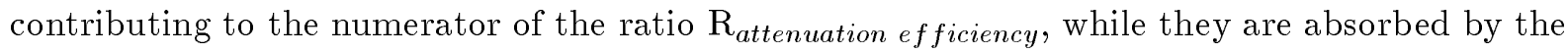
delimiter if no collimator is present. For very thick collimators, however (see the 4.5 -cm-thick case), the higher neutron leakage through the delimiter due to the presence of the thick collima- 
tor is not compensated by the increase in neutron current in the exit window, and therefore the ratio $\mathrm{R}_{\text {attenuation efficiency }}$ becomes lower. Considering both Figs. $6 \mathrm{a}$ and $6 \mathrm{~b}$, one can conclude that 1.5- and 3.0-cm-thick graphite collimators of angle $20 \mathrm{deg}$ are close to optimal. A 3-cm-thick collimator of angle $20 \mathrm{deg}$ will be used further in this study, because it gives a slightly higher ratio $\mathrm{R}_{\text {exit window }}$ in Fig. 6a. The same work has been repeated for lead instead of graphite for the collimator and front-reflector. The results were very similar and lead could easily substitute for graphite, in case photon production in the moderator is of concern.

\section{III.4 Methodology for the optimization of the BSAs}

The quantities used to measure the quality of the neutron beam coming out of the BSA are those defined in Sec. II: the ratio $D_{\text {syn }} / D_{\text {bone }}$ of the absorbed synovium dose to the average absorbed bone dose, the ratio $D_{s y n} / D_{s k}$ of the absorbed synovium dose to the maximum absorbed front skin dose, the absorbed synovium dose $\mathrm{N} \cdot \mathrm{D}_{\text {syn }}$, and the treatment time $\mathrm{T}$. Several parameters can be varied for the optimization, the materials $\mathrm{M}_{i}$ of the moderator, the number I of different materials, the material thicknesses $\mathrm{Th}_{i}$, the moderator diameter $\mathrm{D}$, the sideand back-reflector materials $\mathrm{M}_{\text {side-refl }}$ and $\mathrm{M}_{\text {back-refl }}$, the side- and back-reflector thicknesses $\mathrm{Th}_{\text {side-refl }}, \mathrm{Th}_{\text {back-refl }}$, etc. The delimiter and front-reflector do not need to be included in this optimization, as they have been optimized in Sec. III.3. Even though one could allow the variable parameters to vary to infinity, one will set limits on their ranges. Because of weight concerns, the moderator diameter $\mathrm{D}$ will vary in the range 0 to $100 \mathrm{~cm}$, and the side- and back- reflector thicknesses $\mathrm{Th}_{\text {side-refl }}$ and $\mathrm{Th}_{\text {back-refl }}$ in the range 0 to $50 \mathrm{~cm}$. The number of materials I in the moderator will be limited to 3 . The reflector materials $\mathrm{M}_{\text {refl }}$, as well as the neutron moderating materials $\mathrm{M}_{i}$, will be chosen based on the work done in Ref. [12] for BNCT. Let $\vec{x}$ be a vector containing all the parameters. The optimal BSA will be such that the set of 
parameters gives the highest therapeutic ratio $\mathrm{D}_{\text {syn }} / \mathrm{D}_{\text {bone }}$

$$
\frac{D_{\text {syn }}}{D_{\text {bone }}}\left(\vec{x}_{\text {optimal }}\right)=\max \left(\frac{D_{\text {syn }}}{D_{\text {bone }}}(\vec{x})\right)
$$

for all possible $\vec{x}$ under the following contraints:

$$
T\left(\vec{x}_{\text {optimal }}\right) \leq T_{\max }
$$

for the treatment time $\mathrm{T}$, and

$$
\frac{D_{\text {syn }}}{D_{\text {sk }}}\left(\vec{x}_{\text {optimal }}\right) \geq 12.5
$$

for the therapeutic ratio absorbed synovium dose to absorbed maximum front skin dose. The treatment time $\mathrm{T}$ is determined using Eq. (2), in which the number of source neutrons $\mathrm{N}$ is determined using Eq. (1). The neutron source strength $\mathrm{S}$ depends on the fusion reaction considered. In this article, we will use $7 \times 10^{11} \mathrm{~s}^{-1}$ for D-D (see Sec. III.5) and $10^{14} \mathrm{~s}^{-1}$ for D-T (see Sec. III.6). The maximum permissible treatment time $\mathrm{T}_{\max }$ is set to $10 \mathrm{~min}$.

With 3 materials in the moderator, the number of independent variables is 11 . The ratio $D_{\text {syn }} / D_{\text {bone }}(\vec{x})$ is an 11-dimensional hypersurface imbedded in a 12-dimensional hyperspace. Because of the complexity of this optimization problem, one has to make some assumptions. Let $\vec{x}_{s u b}$ be the set of parameters $\vec{x}$ minus its $\mathrm{M}_{\text {side-refl }}$ and $\mathrm{Th}_{\text {side-refl }}$ components. One will assume that if the side-reflector material $\mathrm{M}_{\text {side-refl }}$ and thickness $\mathrm{Th}_{\text {side-refl }}$ are optimal for one subset $\vec{x}_{s u b}$ of parameters, they will be optimal for all subsets $\vec{x}_{s u b}$ of parameters. The same assumption will be made for the back-reflector material $\mathrm{M}_{\text {back-refl }}$ and thickness $\mathrm{Th}_{\text {back-refl }}$, as well as for the moderator diameter D. With these assumptions, one can optimize (a) the side-reflector material and thickness, (b) the back-reflector material and thickness, and (c) the moderator diameter, without optimizing simultaneously the other parameters. Once the ratio $D_{\text {syn }} / D_{\text {bone }}(\vec{x})$ is maximized in terms of these parameters, the optimization problem is reduced to finding the maximum of a 6-dimensional hypersurface in a 7-dimensional hyperspace. Furthermore, in light of our work for BNCT, we will limit the number of moderating materials 
to just a few: heavy water for D-D neutrons, lead, lithium fluoride, and heavy water (in this order) for D-T neutrons. The optimization problem reduces then to finding the maximum of a line in a 2-dimensional plane for $\mathrm{D}$-D, and the maximum of a 3-dimensional hypersurface imbedded in a 4-dimensional hyperspace for $\mathrm{D}$ - $\mathrm{T}$. The optimization must be performed with the aforementioned constraints.

\section{III.5 Moderation of D-D neutrons}

First, a generic moderator is used to analyze the impact of the reflector material, the reflector thickness, and the moderator diameter. The quantities used to measure the neutron beam quality are the treatment time and the therapeutic ratios. Then, different combinations of materials are considered, with the goal of producing an intense thermal neutron beam with the fast neutron component reduced to a minimum level.

It is worth briefly examining the neutron cross sections of heavy water. The data are taken from the MCNP (Ref. [9]) cross-section library. As shown in Fig. 7, heavy water has a high elastic scattering cross section and a very low absorption cross section at low energies. Its moderating ratio is very high in that range. However, the elastic scattering cross section of

deuterium decreases at energies higher than $2 \mathrm{MeV}$. It decreases by approximately a factor 4 between 2.45 and $14.1 \mathrm{MeV}$. Moreover, the absorption cross section of oxygen increases steeply at $3 \mathrm{MeV}$. Therefore, the moderating ratio - defined as $\xi \Sigma_{s} / \Sigma_{a}$ - of heavy water for D-T source neutrons will be much lower than for D-D source neutrons. This fact will have some important consequences for the D-T neutron moderation and will be discussed further in Sec. III.6.

The generic BSA used for the BSA optimization in Secs. III.5.1 through III.5.4 is identical to the one described in Sec. III.2 and shown in Fig. 3. However, the front-reflector and the delimiter between the heavy water moderator and the knee, as well as the collimator, are different. As described in Sec. III.3, the front-reflector is a 5-cm-thick graphite layer and is followed 
by a 8 -cm-thick lithiated polyethylene delimiter. The collimator is a 3 -cm-thick graphite layer at an angle of $20 \mathrm{deg}$. A 2-cm-thick layer of lithiated polyethylene (see Fig. 2) is left between the collimator and the knee in order to compensate for the lower neutron attenuation of the collimator material. A D-D neutron source strength of $7 \cdot 10^{11} \mathrm{~s}^{-1}$ is assumed at the target. This strength corresponds to a neutron generator in which a 1.5-A mainly monatomic deuteron beam of energy $150 \mathrm{keV}$ hits a large area $T i D_{1.6}$ target (taken from Ref. [15]). Unless specified otherwise, this delimiter, collimator, and neutron source strength will be used further in the BSA optimization for D-D neutrons.

Figures $8 \mathrm{a}$ and $8 \mathrm{~b}$ show a comparison between the therapeutic ratios and treatment times obtained using the full-simulation and the dose-response database simulation methods for different graphite side-reflector thicknesses. The comparison shows an overall good agreement between the two simulation methods. The agreement between the trendlines shows that the dose-response database simulation method can be used for the optimization of the BSA, since the maxima and minima occur for the same side-reflector thicknesses. The good agreement between the values confirms the fact that most particles reaching the knee are transmitted through the exit window of the BSA, as opposed to leaking through the delimiter.

\section{III.5.1 Side-reflector design}

The side-reflector material and thickness are varied to see the impact of these two parameters on the treatment time and therapeutic ratios. Three materials are used for the simulations: graphite, lead, and aluminium oxide $\mathrm{Al}_{2} \mathrm{O}_{3}$. The side-reflector thicknesses range from 0 to 50 $\mathrm{cm}$. The BSA is otherwise the same as the one described in Sec. III.5. The results of this simulation study are presented in Figs. 9a and 9b. Graphite gives the highest therapeutic ratios for all side-reflector thicknesses, while aluminum oxide gives the lowest. A more detailed analysis has been carried out to explain these effects. Aluminum oxide generates more photons than 
graphite and lead when bombarded by neutrons. The excessive photon dose in the knee results in lower therapeutic ratios. Concerning lead, it has a lower moderating power than graphite. Therefore, more fast neutrons are reflected from the lead than from the graphite reflector. By itself and by photon induction in the tissues, this higher fast neutron component in the neutron beam decreases the therapeutic ratios. The therapeutic ratios increase asymptotically with the reflector thickness, to reach plateaus at $\sim 30 \mathrm{~cm}$. The treatment time decreases very rapidly with reflector thicknesses up to $30 \mathrm{~cm}$. Graphite has the shortest treatment times for all sidereflector thicknesses. As a conclusion, a 30-cm-thick graphite side-reflector will be used for the simulations in Secs. III.5.2, III.5.3, and III.5.4.

\section{III.5.2 Back-reflector design}

With the material and thickness of the side-reflector determined in Sec. III.5.1, we analyze the effect of the back-reflector material and thickness. The same materials and thicknesses as in Sec. III.5.1 are considered for the back-reflector. Figures 10a and 10b show the results of this parametric study. The back-reflector thickness has no significant influence on the therapeutic ratios, while the treatment time decreases rapidly for back-reflector thicknesses up to $30 \mathrm{~cm}$. Concerning the back-reflector material, graphite exhibits better characteristics than lead and aluminum oxide. A 30-cm-thick layer of graphite will be used further in Secs. III.5.3 and III.5.4 for the back-reflector.

\section{III.5.3 Optimization of moderator thickness}

With the 30-cm-thick graphite side- and back-reflectors, simulations have been carried out with different heavy water moderator thicknesses, from 0 to $100 \mathrm{~cm}$. The results are shown in Fig. 11. Both therapeutic ratios increase with the thickness for thicknesses up to $70 \mathrm{~cm}$. For larger thicknesses, the ratio $\mathrm{D}_{\text {syn }} / \mathrm{D}_{\text {bone }}$ decreases slightly, while the ratio $\mathrm{D}_{\text {syn }} / \mathrm{D}_{\text {sk }}$ remains constant. The treatment time increases exponentially with the thickness. A 50-cm-thick heavy water 
moderator gives simultaneously high therapeutic ratios and reasonable treatment times. This thickness will be used for the moderator diameter parametric study. Other materials were tried for the moderation, but none of them had better characteristics than heavy water, and they were not retained.

\section{III.5.4 Optimization of moderator diameter}

The moderator diameter, which had been set to $35 \mathrm{~cm}$ initially and remained unchanged throughout the previous sections, was then varied in the range 15 to $75 \mathrm{~cm}$; the results are shown in Fig. 12. The therapeutic ratios increase steeply up to a diameter of $35 \mathrm{~cm}$; after that, the gains are less pronounced. The treatment time decreases with increasing diameter. A diameter of 55 $\mathrm{cm}$ is considered optimal for both the treatment time and the therapeutic ratios.

To summarize the results obtained in the BSA optimization study, we conclude that a 50-cm-thick, 55-cm-diam heavy water moderator is optimal for the moderation of D-D neutrons. For the reflection of neutrons leaking out of the moderator, the heavy water is surrounded by a 30-cm-thick graphite side-reflector and preceded by a 30-cm-thick graphite back-reflector. Concerning the delimiter and the front-reflector, an 8-cm-thick lithiated polyethylene delimiter reduces sufficiently radiation exposure to the patient organs other than the knee, while a 5 -cmthick graphite front-reflector located between the heavy water and the delimiter reflects neutrons leaking out of the heavy water volume back to this volume and reduces neutron losses by neutron absorption in the delimiter. A 3-cm-thick graphite collimator at an angle of $20 \mathrm{deg}$ is optimal to collimate the neutrons through the exit window. A 2-cm-thick layer of lithiated polyethylene is left between the collimator and the knee to compensate for the lower neutron attenuation of the collimator material.

With this BSA, a full simulation has been performed, and therapeutic ratios of $\mathrm{D}_{\text {syn }} / \mathrm{D}_{\text {bone }}=$ $396 \pm 4$ and $\mathrm{D}_{s y n} / \mathrm{D}_{s k}=113 \pm 2$ were obtained. These ratios can be compared with the 
ones obtained using the dose-response database simulation method, $\mathrm{D}_{\text {syn }} / \mathrm{D}_{\text {bone }}=407 \pm 2$ and $\mathrm{D}_{\text {syn }} / \mathrm{D}_{s k}=122 \pm 1$. With a D-D neutron source strength of $7 \cdot 10^{11} \mathrm{~s}^{-1}$, one can predict a treatment time of $2 \mathrm{~h}$ and $20 \mathrm{~min}$. Unless this long treatment time is reduced by an increase of the ${ }^{10} \mathrm{~B}$ concentration in the synovium, or by a neutron flux amplifier such as a fission plate converter, it will be a major obstacle for the use of the D-D reaction for BNCS.

\section{III.6 Moderation of D-T neutrons}

The $\mathrm{D}$ - $\mathrm{T}$ reaction was considered next because its neutron yield is more than one order of magnitude higher than D-D. Therefore, shorter treatment times can be expected using this reaction. The neutron yield at the target used in this study is $10^{14} \mathrm{~s}^{-1}$ and was taken from Ref. [15]. It corresponds to the neutron yield of a mainly monatomic 2-A mixed deuteron/triton beam bombarding titanium or scandium targets with an energy of $150 \mathrm{keV}$.

The first moderator tried for the moderation of D-T neutrons was similar to the one used for the D-D neutrons and was composed of a single layer of heavy water. Even with thick layers of heavy water, this moderator did not result in good therapeutic ratios. The reason for this is shown in Fig. 7; the elastic scattering cross section of heavy water for $14.1-\mathrm{MeV}$ neutrons is relatively low. An analysis of the neutron energy distribution after moderation of D-T neutrons by heavy water reveals the presence of two peaks, one thermal peak and one peak at energies $>10 \mathrm{MeV}$. Other materials were thus considered for moderation.

Neutron moderation for BNCT [12] showed that lead, bismuth, iron, lithium fluoride enriched in the isotope ${ }^{7} \mathrm{Li}$, and FLUENTAL C [16] (mixture of $40 \%$ aluminium and $60 \%$ aluminium fluoride) used for moderation of D-D and D-T neutrons gave good neutron spectra for BNCT applications. Lead, bismuth, and iron were particularly good for reducing the fast components of the neutron spectrum. Based on this study, a new moderator was designed for the moderation of $14-\mathrm{MeV}$ neutrons. It is composed of a $20-\mathrm{cm}$-thick layer of lead, followed by 
a 25 -cm-thick layer of lithium fluoride ${ }^{7} \mathrm{LiF}$, a 45 -cm-thick layer of heavy water, and a 1 -mmthick layer of lead to attenuate the photon flux at the end of the moderation. The moderator diameter was initially set to $35 \mathrm{~cm}$. The side- and back-reflectors are 10-cm-thick layers of lead. Ten-centimetre-thick lithiated polyethylene layers surround the side-reflector and precede the back-reflector. The front-reflector, the collimator, and the delimiter between the moderator and the knee are identical to the ones described in Sec. III.5.

Figures $13 \mathrm{a}$ and $13 \mathrm{~b}$ compare the therapeutic ratios and treatment times obtained using the full-simulation and the dose-response database simulation methods for different graphite sidereflector thicknesses, using a 5-cm-thick lead front-reflector and a 3-cm-thick lead collimator. Figures $14 \mathrm{a}$ and $14 \mathrm{~b}$ show the same comparison but using graphite instead of lead for the frontreflector and the collimator. There is an overall good agreement between the trendlines shown in the figures for the two simulation methods. This demonstrates that the dose-response database simulation method can be used for the optimization of BSAs for D-T neutrons. In general, the dose-response database simulation method gives higher therapeutic ratios and shorter treatment times than the full-simulation method. The lead front-reflector gives slightly higher $(\sim 5 \%)$ therapeutic ratios than the graphite one for side-reflector thicknesses between 10 and $30 \mathrm{~cm}$. Therefore, a lead front-reflector will be used further in Secs. III.6.1 through III.6.4 for the BSA optimization. Since the graphite front-reflector gives approximately the same results as the lead front-reflector, it could easily replace it without affecting significantly the BSA performance in case the front-reflector weight is of concern.

\section{III.6.1 Side-reflector design}

Using the lead front-reflector, the side-reflector material and thickness are optimized. The materials considered for reflection are the same as the ones used for the D-D neutrons: lead, graphite, and aluminum oxide. The results of this optimization are shown in Figs. 15a and 15b. 
The therapeutic ratios increase and the treatment time decreases asymptotically with the thickness of the side-reflector. Aluminum oxide as a reflector results in very low therapeutic ratios due to the significant gamma-ray production in this material, which contaminates the neutron beam to be used for treatment. The highest therapeutic ratios and shortest treatment times are obtained with the lead side-reflector. Large thicknesses of lead are not necessary for high therapeutic ratios, $90 \%$ of the asymptotic values of the therapeutic ratios can be obtained using a 10 -cm-thick lead side-reflector. Graphite gives therapeutic ratios $\sim 10 \%$ lower than lead for thicknesses larger than $30 \mathrm{~cm}$. A 30-cm-thick graphite side-reflector and a 10-cmthick lead side-reflector give identical therapeutic ratios. However, since the density of lead is approximately seven times higher than the density of graphite, the weight increase occuring when lead is used instead of graphite is not justified even though the side-reflector thickness is smaller. A 30-cm-thick graphite side-reflector will therefore be used further in this study. We keep in mind however that therapeutic ratios higher by $\sim 10 \%$ could be obtained using heavy lead side-reflectors.

\section{III.6.2 Back-reflector design}

A brief analysis of the back-reflector material and thickness was also performed. The results are plotted in Figs. 16a and 16b, where the therapeutic ratios and treatment times, respectively, are plotted as a function of the back-reflector thickness. The ratios are basically constant over all thicknesses, while the treatment time decreases asymptotically with the thickness. Graphite is again preferred over other materials due to its lower density. A 30-cm-thick layer of graphite will be used further for the optimization of the moderator diameter and thickness.

\section{III.6.3 Optimization of moderator diameter}

With the side- and back-reflectors described in Secs. III.6.1 and III.6.2, a moderator diameter parametric study is performed. The moderator diameter is varied from 15 to $85 \mathrm{~cm}$ in increments 
of $10 \mathrm{~cm}$. Both $30-\mathrm{cm}$-thick layers of lead and graphite were considered as side-reflectors for this study, since both materials gave similar results in the side-reflector thickness parametric study. The results of this study are shown in Fig. 17. The therapeutic ratios increase substantially with the moderator diameter, and the treatment time decreases significantly. In the case of the graphite reflector, for instance, the two therapeutic ratios $\mathrm{D}_{\text {syn }} / \mathrm{D}_{\text {sk }}$ and $\mathrm{D}_{\text {syn }} / \mathrm{D}_{\text {bone }}$ increase from 89 and 234 for a 35 -cm-diam moderator to 108 and 308 for a 55 -cm-diam moderator, and to 112 and 338 for a 85 -cm-diam moderator. For large moderator diameters, the difference between the graphite and the lead reflectors is not very pronounced, 5 to $10 \%$.

\section{III.6.4 Optimization of moderator composition and thickness}

For the moderator thickness and composition study, a 55-cm-diam moderator with a graphite reflector will be used. One could obtain larger therapeutic ratios and better treatment times with even larger moderators, but $55 \mathrm{~cm}$ is a good compromise between moderator volume, and thus weight and moderator efficiency in terms of therapeutic ratios.

So far, the moderator was composed of a 20-cm-thick layer of lead, followed by a 25cm-thick layer of lithium fluoride ${ }^{7} \mathrm{LiF}$, a 45 -cm-thick layer of heavy water, and a 1 -mm-thick layer of lead to attenuate the photon flux at the end of the moderation. To see whether higher therapeutic ratios and shorter treatment times can be obtained with a better moderator, a parametric study was performed using the aforementioned moderator as a reference moderator and changing the lead, lithium fluoride, and heavy water thick-layer thicknesses by increments of $5 \mathrm{~cm}$. Iron was also tried as a moderator material, but the high-energy photon production of this material did not make it attractive.

Figures $18 \mathrm{a}$ and $18 \mathrm{~b}$ show the therapeutic ratios and treatment times, respectively, as a function of the moderator material thickness increment. The vertical line is the reference moderator. The first data points of the lead curves represent the case of a moderator without 
lead. We will first examine the influence of lead in the moderation. The treatment time decreases when a 5-cm-thick layer of lead is added to the moderator without lead. This illustrates the effect of the $(n, 2 n)$ reactions occuring in lead at high neutron energies.

Therapeutic ratios increase steeply for lead layer thicknesses up to $25 \mathrm{~cm}$ and reach asymptotically a plateau for larger thicknesses. From the therapeutic ratios plotted in Fig. 18a, it appears that an additional 5 -cm-thick layer of lead would be beneficial for the treatment.

Concerning heavy water, the therapeutic ratios do not increase as rapidly with the thickness increment as with lead or lithium fluoride. For very large heavy water thicknesses, however, the therapeutic ratios reach values $5 \%$ higher than the two other materials. The behavior of lithium fluoride is somewhere between the behavior of lead and that of heavy water. From the observations made in Figs. 18a and 18b, it appears that replacing $5 \mathrm{~cm}$ of heavy water by $5 \mathrm{~cm}$ of lead would be beneficial for both the therapeutic ratios and the treatment times.

A simulation run was performed with $25 \mathrm{~cm}$ of lead, $25 \mathrm{~cm}$ of lithium fluoride, and 40 $\mathrm{cm}$ of heavy water. The therapeutic ratios were effectively higher and equal to $\mathrm{D}_{s y n} / \mathrm{D}_{s k}=$ $111.9 \pm 1.9$ and $\mathrm{D}_{\text {syn }} / \mathrm{D}_{\text {bone }}=324.6 \pm 3.3$, respectively. The treatment time decreases slightly from $4.78 \pm 0.03 \mathrm{~min}$ to $4.72 \pm 0.05 \mathrm{~min}$.

A full simulation was then carried out to check the accuracy of these results. The therapeutic ratios for the full-simulation run were $\mathrm{D}_{\text {syn }} / \mathrm{D}_{s k}=97.9 \pm 1.6$ and $\mathrm{D}_{\text {syn }} / \mathrm{D}_{\text {bone }}=$ $293.6 \pm 3.4$, while the treatment time was $5.74 \pm 0.04 \mathrm{~min}$. The 10 to $15 \%$ overestimation of the therapeutic ratios and $20 \%$ underestimation of the treatment time obtained with the doseresponse database simulation method are in good agreement with the differences observed in Figs. 13a and 13b and confirm that a full simulation is always necessary, after the BSA has been optimized with the dose-response database simulation method. A full-simulation run was then performed with lead back- and side-reflectors instead of graphite ones, since we had noticed in Sec. III.6.1 that this would enhance the therapeutic ratios and reduce the treatment time. The 
therapeutic ratios obtained were $\mathrm{D}_{\text {syn }} / \mathrm{D}_{s k}=111.0 \pm 2.4$ and $\mathrm{D}_{\text {syn }} / \mathrm{D}_{\text {bone }}=323.2 \pm 6.1$, while the treatment time was $4.03 \pm 0.04 \mathrm{~min}$. These treatment characteristics are $\sim 10 \%$ better than the ones for the graphite reflectors. Depending on whether weight is of concern, lead or graphite will be selected as a reflector material.

In summary, the best moderator that the authors tried was a 25 -cm-thick layer of lead followed by a 25 -cm-thick layer of lithium fluoride ${ }^{7} \mathrm{LiF}$ and a 40 -cm-thick layer of heavy water. This moderator is $55 \mathrm{~cm}$ in diameter and surrounded by a 30-cm-thick graphite layer to reflect neutrons that would otherwise leak out of the moderator. To reduce radiation exposure to the patient's organs other than the knee, a 8-cm-thick lithiated polyethylene delimiter is placed between the moderator and the patient's knee. A 5-cm-thick lead front-reflector is placed between the moderator and the delimiter to reflect neutrons leaking out through this surface. A 3-cm-thick lead collimator at an angle of $20 \mathrm{deg}$ is used to collimate the neutrons to the knee. With this BSA, therapeutic ratios of $\mathrm{D}_{\text {syn }} / \mathrm{D}_{s k}=97.9 \pm 1.6$ and $\mathrm{D}_{\text {syn }} / \mathrm{D}_{\text {bone }}=293.6 \pm 3.4$ were obtained; the treatment time was $5.74 \pm 0.04 \mathrm{~min}$. Lead back- and side-reflectors give in general $\sim 10 \%$ better results but are much heavier.

\section{CONCLUSION}

The neutron sources considered for BNCS were D-D and D-T, which emit 2.45 and $14.1 \mathrm{MeV}$ neutrons. The neutron yields considered for the D-D and D-T reactions were $7 \cdot 10^{11}$ and $10^{14}$ $\mathrm{s}^{-1}$, respectively. The dose-response database simulation method developed in a previous paper was used to accelerate the design of BSAs for BNCS. Good agreement was observed between this method and the more accurate method simulating the entire geometry at once with MCNP. A 50-cm-thick, 55-cm-diam heavy water moderator surrounded by a graphite reflector was found to be optimal for moderation of D-D neutrons. Table II shows the description of the optimal BSA for D-D. As we can observe in the table, the treatment time is longer than $2 \mathrm{~h}$. It could 
potentially be reduced if the ${ }^{10} \mathrm{~B}$ concentration in the synovium was increased. For D-T neutrons, a 25 -cm-thick layer of lead followed by a 25 -cm-thick layer of lithium fluoride ${ }^{7} \mathrm{LiF}$ and a 40 $\mathrm{cm}$-thick layer of heavy water gives high therapeutic ratios and a treatment time of $\sim 5$ min. In the case of $\mathrm{D}-\mathrm{T}$, an increase in the moderator assembly diameter significantly reduced the treatment time.

\section{ACKNOWLEDGEMENTS}

This work is partly supported by the U.S. Department of Energy under contract DE-AC03$76 \mathrm{SF} 00098$. 


\section{List of Tables}

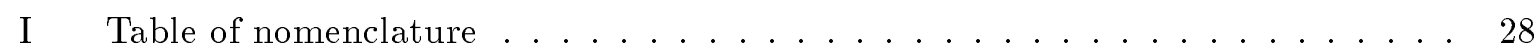

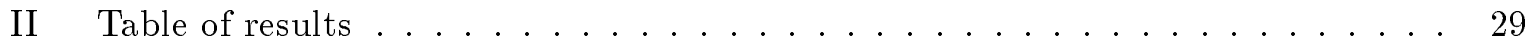




\section{List of Figures}

1 Cross-section view of the cylindrical knee model used in the MCNP simulations. Absorbed doses are computed as a function of the depth within the 2.5-cm-diam cylinder (represented by the two horizontal lines) intersecting the different tissue

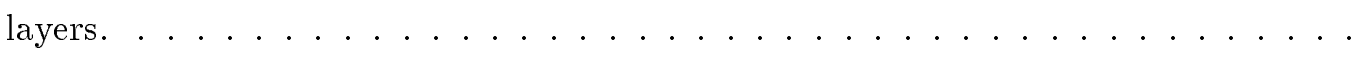

2 BSA showing the 8.7-cm-diam exit window where the neutron and photon spectra are calculated, referred to as exit window throughout the text. The collimator is the truncated cone of angle of aperture $\alpha$ located between the moderator and the

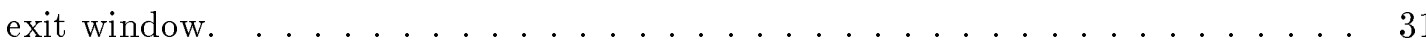

3 Generic BSA used for the optimization. . . . . . . . . . . . . . 32

4 Ratios (a) $\mathrm{R}_{\text {exit window }}$ and (b) $\mathrm{R}_{\text {attenuation efficiency }}$ as a function of the delimiter/frontreflector thickness and material. . . . . . . . . . . . . . . 33

$5 \quad$ Neutron beam directionality - measured by the ratio of the neutron current $J_{+}$to the neutron flux $\phi$ across the exit window — as a function of the delimiter/frontreflector thickness and material. . . . . . . . . . . . . .

6 Ratios (a) $R_{\text {exit window }}$ and (b) $R_{\text {attenuation efficiency }}$ as a function of the graphite collimator angle and thickness. The front-reflector is a 5 -cm-thick graphite layer and is followed by a 8 -cm-thick lithiated polyethylene delimiter. . . . . . . . . 35

7 Macroscopic cross sections of heavy water. . . . . . . . . . . . 36

8 Comparison of (a) therapeutic ratios and (b) treatment times obtained with the dose-response database simulation and the full simulation methods. . . . . . . .

9 (a) Therapeutic ratios and (b) treatment time as a function of the side-reflector

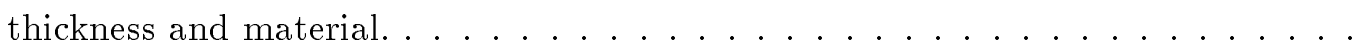

10 (a) Therapeutic ratios and (b) treatment time as a function of the back-reflector thickness and material. . . . . . . . . . . . . . . 
11 Therapeutic ratios and treatment time versus heavy water moderator thickness. . 40

12 Therapeutic ratios and treatment time versus heavy water moderator diameter. . 41

13 Comparison of (a) therapeutic ratios and (b) treatment times obtained with the dose-response database simulation and the full simulation methods using a lead front-reflector and a lead collimator. . . . . . . . . . . . . . 42

14 Comparison of (a) therapeutic ratios and (b) treatment times obtained with the dose-response database simulation and the full simulation methods using a graphite front-reflector and a graphite collimator. . . . . . . . . . .

15 (a) Therapeutic ratios and (b) treatment time as a function of the side-reflector thickness and material. . . . . . . . . . . . . . .

16 (a) Therapeutic ratios and (b) treatment time as a function of the back-reflector thickness and material. . . . . . . . . . . . . . . .

17 Therapeutic ratios and treatment time as a function of the moderator diameter and side-reflector material. A side-reflector thickness $\mathrm{Th}_{\text {side-refl }}$ of $30 \mathrm{~cm}$ is used for all simulations. . . . . . . . . . . . . . . . . .

18 (a) Therapeutic ratios and (b) treatment time as a function of moderator material thickness increment. The reference moderator is composed of a 20 -cm-thick layer of lead, followed by a 25 -cm-thick layer of lithium fluoride ${ }^{7} \mathrm{LiF}$, a 45 -cm-thick layer of heavy water and a 1 -mm-thick layer of lead. . . . . . . . . . 
J.M. Verbeke - Optimization of beam-shaping assemblies for BNCS using the high-energy neutron sources D-D and D-T

\begin{tabular}{|c|c|c|}
\hline Symbol & Definition & Units \\
\hline $\mathrm{D}_{\text {syn }}$ & $\begin{array}{l}\text { average absorbed synovium dose per unit neutron } \\
\text { emitted by the source }\end{array}$ & Gy-equivalent \\
\hline $\mathrm{D}_{s k}$ & $\begin{array}{l}\text { average absorbed front skin dose per unit neutron } \\
\text { emitted by the source }\end{array}$ & Gy-equivalent \\
\hline $\mathrm{D}_{\text {bone }}$ & $\begin{array}{l}\text { average absorbed bone dose per unit neutron } \\
\text { emitted by the source }\end{array}$ & Gy-equivalent \\
\hline $\mathrm{D}_{\text {syn }} / \mathrm{D}_{\text {bone }}$ & $\begin{array}{l}\text { ratio of the absorbed synovium dose to the } \\
\text { absorbed bone dose }\end{array}$ & - \\
\hline $\mathrm{D}_{s y n} / \mathrm{D}_{s k}$ & $\begin{array}{l}\text { ratio of the absorbed synovium dose to the } \\
\text { absorbed front skin dose }\end{array}$ & - \\
\hline $\mathrm{T}$ & treatment time & $\min$ \\
\hline $\mathrm{T}_{\max }$ & maximum permissible treatment time & $\min$ \\
\hline $\mathrm{S}$ & neutron source strength & $\mathrm{s}^{-1}$ \\
\hline $\mathrm{D}$ & moderator diameter & $\mathrm{cm}$ \\
\hline $\mathrm{Th}_{\text {side-refl }}$ & side-reflector thickness & $\mathrm{cm}$ \\
\hline Th $h_{\text {back-refl }}$ & back-reflector thickness & $\mathrm{cm}$ \\
\hline $\begin{array}{l}\mathrm{R}_{\text {exit window }} \\
\text { (see Eq. } 4 \text { ) }\end{array}$ & $\begin{array}{l}\text { ratio of the neutron current across the exit window with } \\
\text { the delimiter/front-reflector to the neutron current across } \\
\text { the exit window without the delimiter/front-reflector }\end{array}$ & - \\
\hline $\begin{array}{l}\mathrm{R}_{\text {attenuation efficiency }} \\
\quad \text { (see Eq. 5) }\end{array}$ & $\begin{array}{l}\text { ratio of the neutron current across the exit window to } \\
\text { the neutron current around the exit window }\end{array}$ & - \\
\hline
\end{tabular}

Table I: Table of nomenclature 
J.M. Verbeke - Optimization of beam-shaping assemblies for BNCS using the high-energy neutron sources D-D and D-T

\begin{tabular}{|c|c|c|c|}
\hline Neutron source & $\mathrm{D}-\mathrm{D}(2.45 \mathrm{MeV})$ & D-T (14.1 MeV) & D-T (14.1 MeV) \\
\hline Neutron source strength $\left[\mathrm{s}^{-1}\right]$ & $7 \times 10^{11}$ & $10^{14}$ & $10^{14}$ \\
\hline $\mathrm{D}_{\text {syn }} / \mathrm{D}_{\text {bone }}[-]$ & $396 \pm 4$ & $293.6 \pm 3.4$ & $323.2 \pm 6.1$ \\
\hline $\mathrm{D}_{s y n} / \mathrm{D}_{s k}[-]$ & $113 \pm 2$ & $97.9 \pm 1.6$ & $111.0 \pm 2.4$ \\
\hline Treatment time [min] & 140 & 5.74 & 4.03 \\
\hline Moderator diameter $[\mathrm{cm}]$ & 55.0 & 55.0 & 55.0 \\
\hline Moderator materials (thicknesses $[\mathrm{cm}]$ ) & $\mathrm{D}_{2} \mathrm{O}(50.0)$ & $\begin{array}{l}\text { lead (25.0) } \\
\mathrm{Li}^{7} \mathrm{~F}(25.0) \\
\mathrm{D}_{2} \mathrm{O}(40.0)\end{array}$ & $\begin{array}{l}\text { lead (25.0) } \\
\mathrm{Li}^{7} \mathrm{~F}(25.0) \\
\mathrm{D}_{2} \mathrm{O}(40.0)\end{array}$ \\
\hline Delimiter material (thickness [cm]) & LiPE $(8.0)$ & LiPE (8.0) & $\operatorname{LiPE}(8.0)$ \\
\hline Front-reflector material (thickness [cm]) & graphite $(5.0)$ & lead $(5.0)$ & lead $(5.0)$ \\
\hline Side-reflector material (thickness $[\mathrm{cm}]$ ) & graphite $(30.0)$ & graphite $(30.0)$ & lead $(30.0)$ \\
\hline Back-reflector material (thickness [cm]) & graphite $(30.0)$ & graphite $(30.0)$ & lead $(30.0)$ \\
\hline Collimator material (thickness $[\mathrm{cm}]$ ) & graphite $(3.0)$ & lead (3.0) & lead (3.0) \\
\hline Collimator angle in degrees & 20 & 20 & 20 \\
\hline
\end{tabular}

Table II: Table of results 
J.M. Verbeke - Optimization of beam-shaping assemblies for BNCS using the high-energy neutron sources D-D and D-T

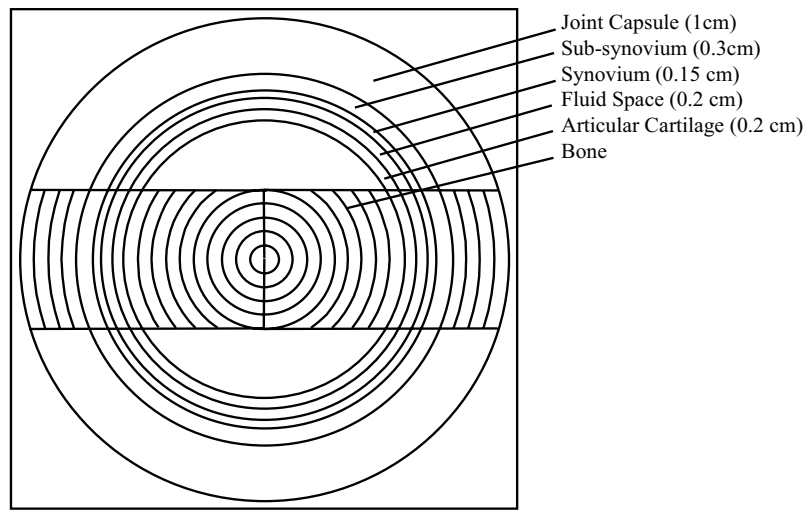

Figure 1: Cross-section view of the cylindrical knee model used in the MCNP simulations. Absorbed doses are computed as a function of the depth within the 2.5-cm-diam cylinder (represented by the two horizontal lines) intersecting the different tissue layers. 
J.M. Verbeke - Optimization of beam-shaping assemblies for BNCS using the high-energy neutron sources D-D and D-T

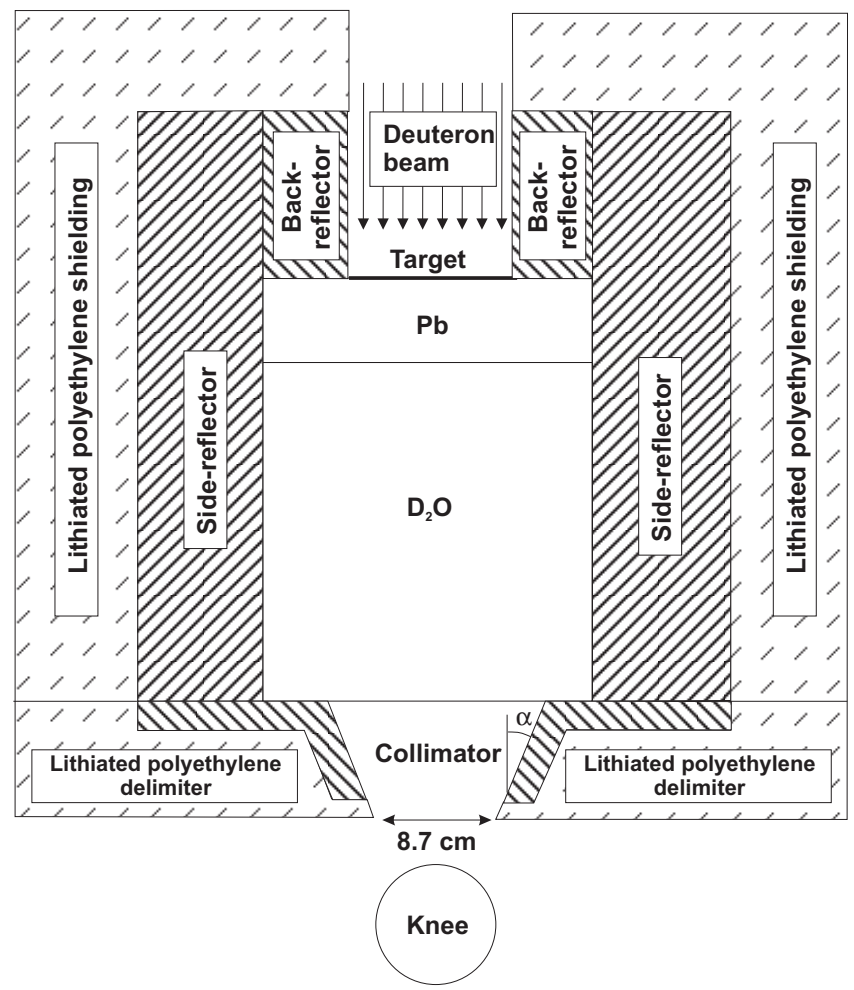

Figure 2: BSA showing the 8.7-cm-diam exit window where the neutron and photon spectra are calculated, referred to as exit window throughout the text. The collimator is the truncated cone of angle of aperture $\alpha$ located between the moderator and the exit window. 
J.M. Verbeke - Optimization of beam-shaping assemblies for BNCS using the high-energy neutron sources D-D and D-T

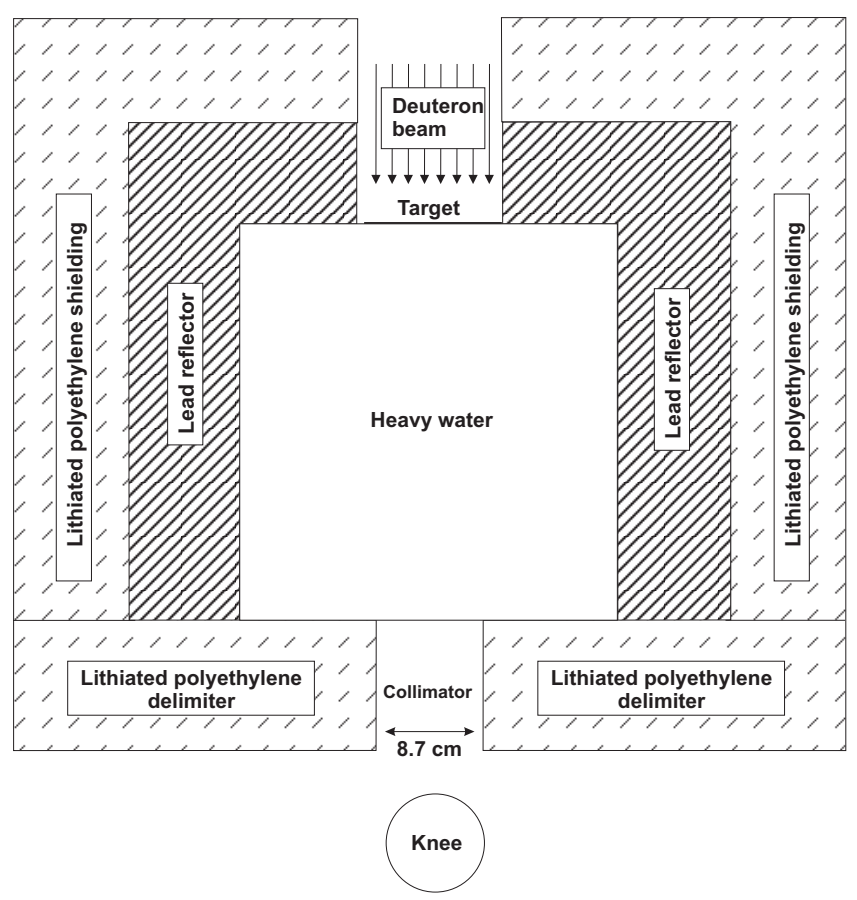

Figure 3: Generic BSA used for the optimization. 
J.M. Verbeke - Optimization of beam-shaping assemblies for BNCS using the high-energy neutron sources D-D and D-T

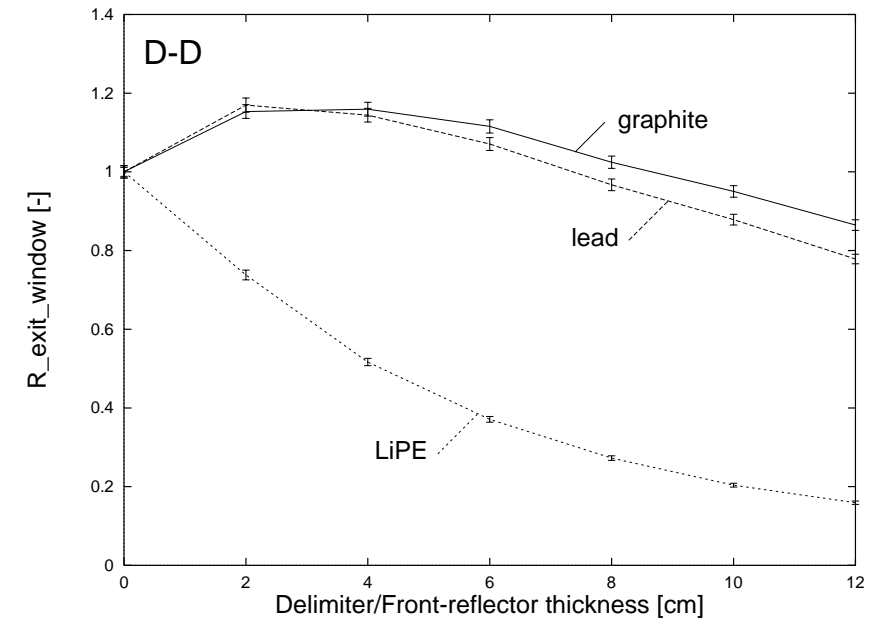

(a)

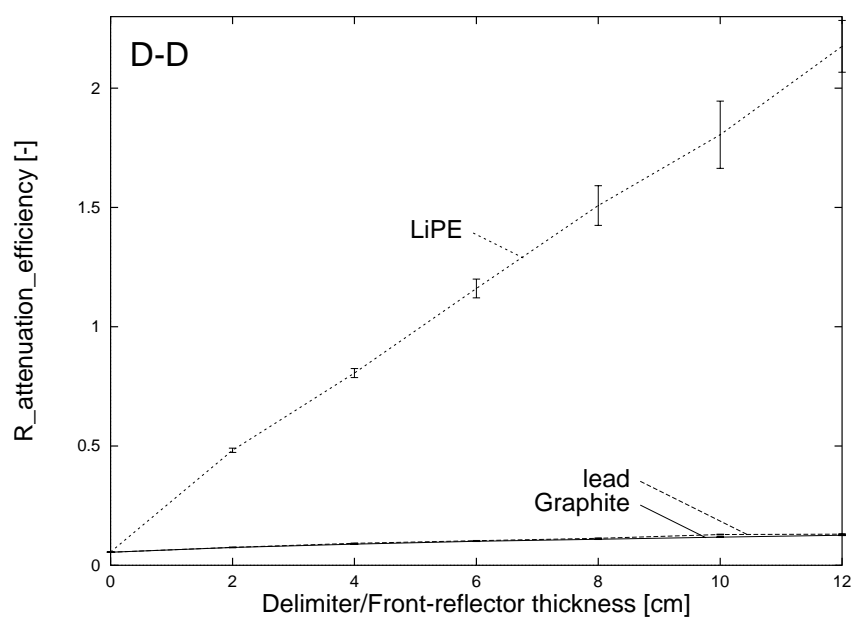

(b)

Figure 4: Ratios (a) $\mathrm{R}_{\text {exit window }}$ and (b) $\mathrm{R}_{\text {attenuation efficiency }}$ as a function of the delimiter/front-reflector thickness and material. 
J.M. Verbeke - Optimization of beam-shaping assemblies for BNCS using the high-energy neutron sources D-D and D-T

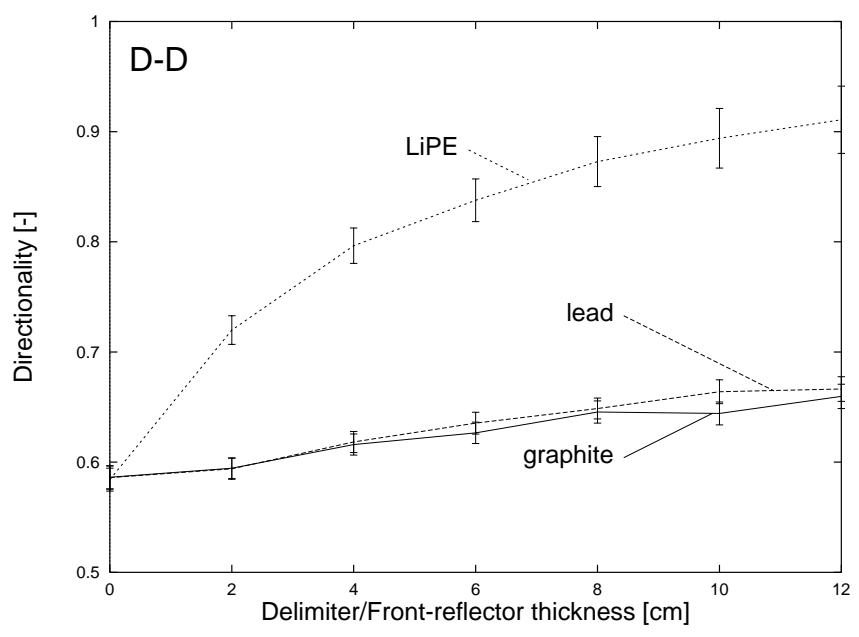

Figure 5: Neutron beam directionality - measured by the ratio of the neutron current $J_{+}$to the neutron flux $\phi$ across the exit window — as a function of the delimiter/front-reflector thickness and material. 
J.M. Verbeke - Optimization of beam-shaping assemblies for BNCS using the high-energy neutron sources D-D and D-T

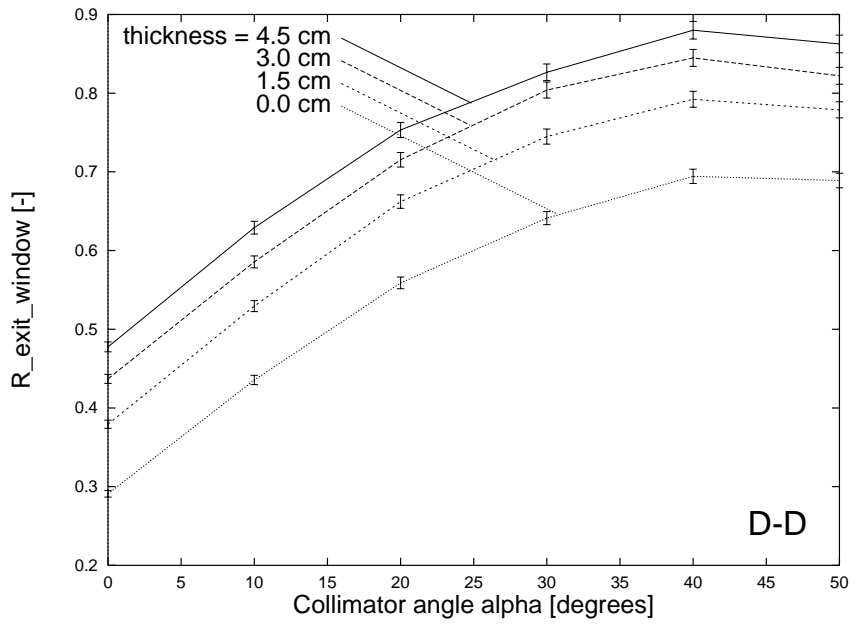

(a)

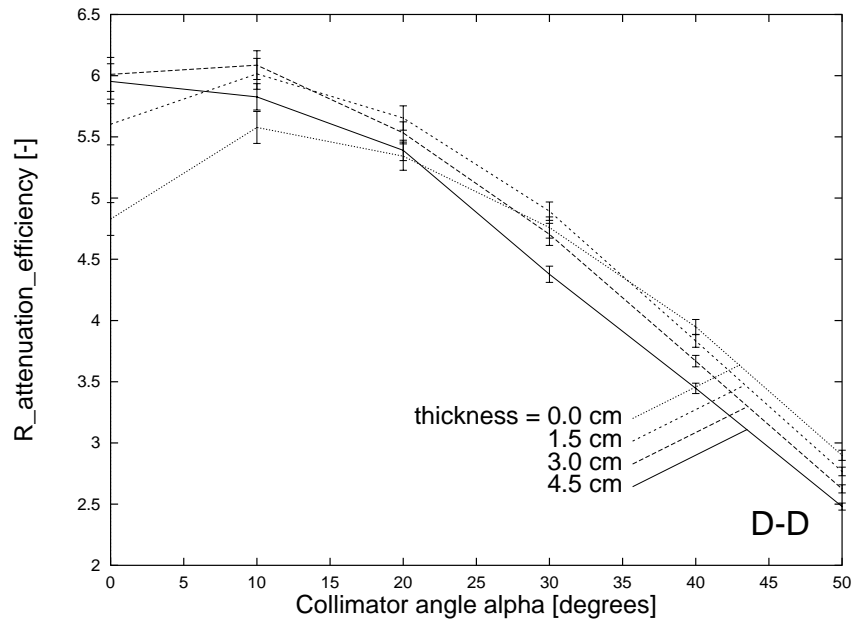

(b)

Figure 6: Ratios (a) $\mathrm{R}_{\text {exit window }}$ and (b) $\mathrm{R}_{\text {attenuation efficiency }}$ as a function of the graphite collimator angle and thickness. The front-reflector is a 5-cm-thick graphite layer and is followed by a 8 -cm-thick lithiated polyethylene delimiter. 
J.M. Verbeke - Optimization of beam-shaping assemblies for BNCS using the high-energy neutron sources D-D and D-T

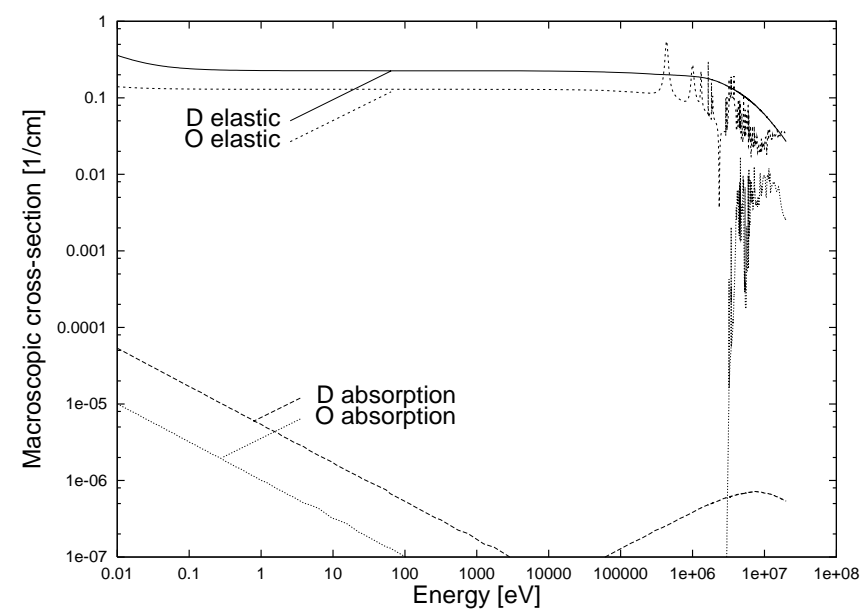

Figure 7: Macroscopic cross sections of heavy water. 
J.M. Verbeke - Optimization of beam-shaping assemblies for BNCS using the high-energy neutron sources D-D and D-T

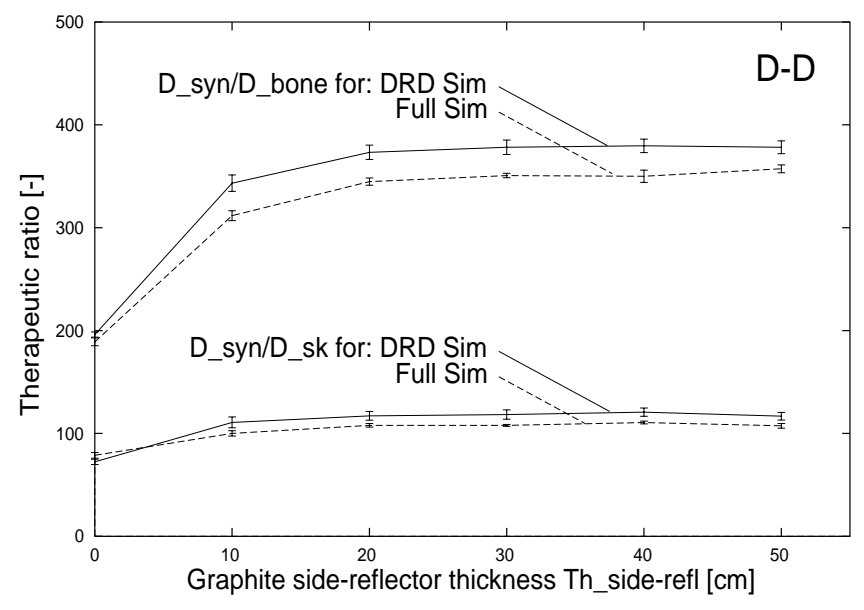

(a)

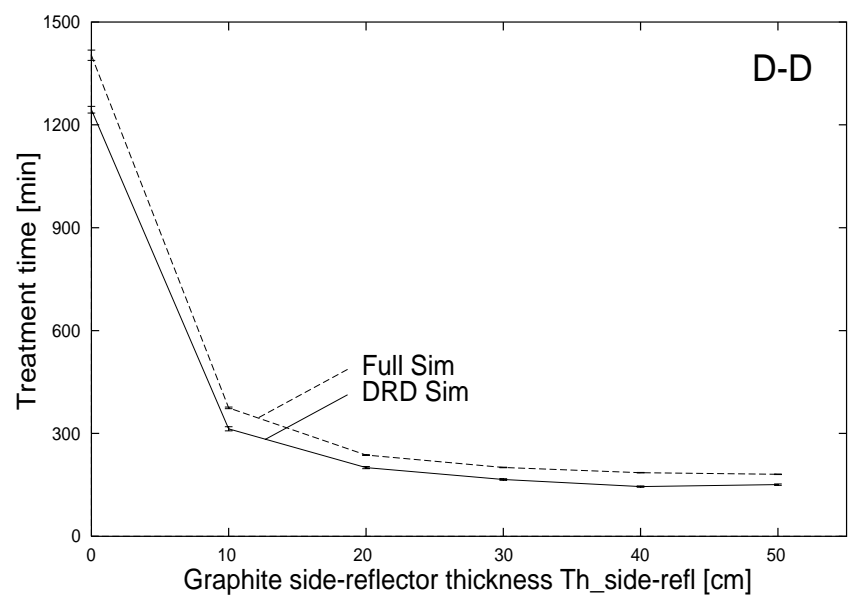

(b)

Figure 8: Comparison of (a) therapeutic ratios and (b) treatment times obtained with the dose-response database simulation and the full simulation methods. 
J.M. Verbeke - Optimization of beam-shaping assemblies for BNCS using the high-energy neutron sources D-D and D-T

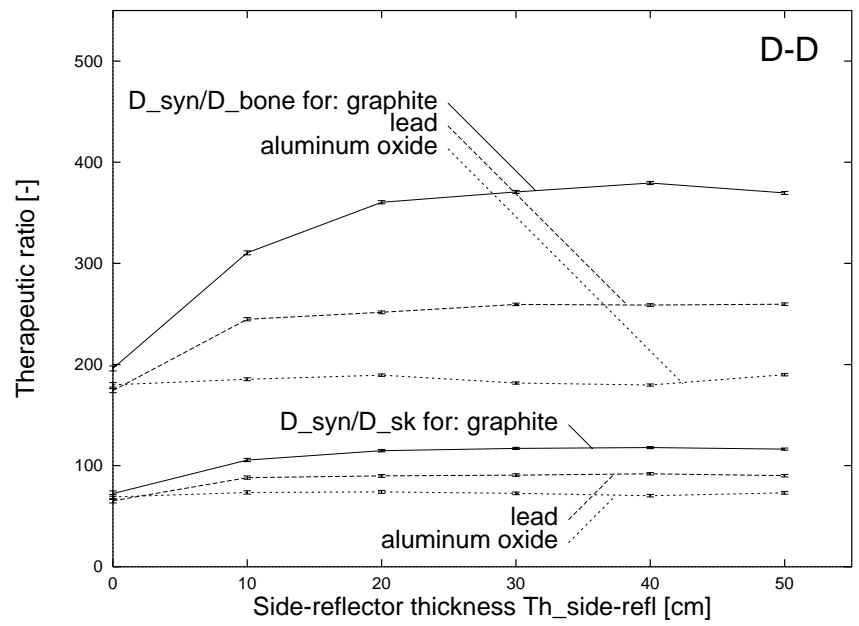

(a)

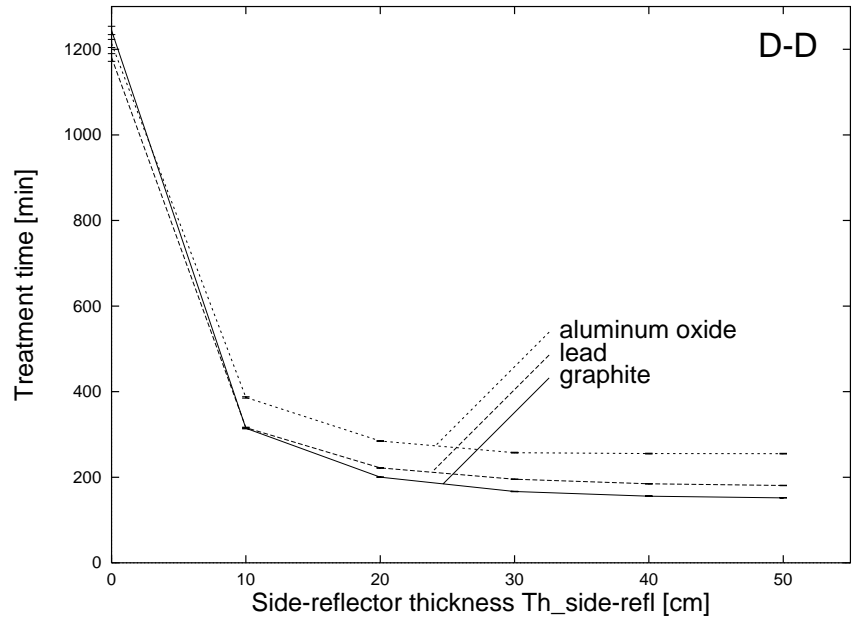

(b)

Figure 9: (a) Therapeutic ratios and (b) treatment time as a function of the side-reflector thickness and material. 
J.M. Verbeke - Optimization of beam-shaping assemblies for BNCS using the high-energy neutron sources D-D and D-T

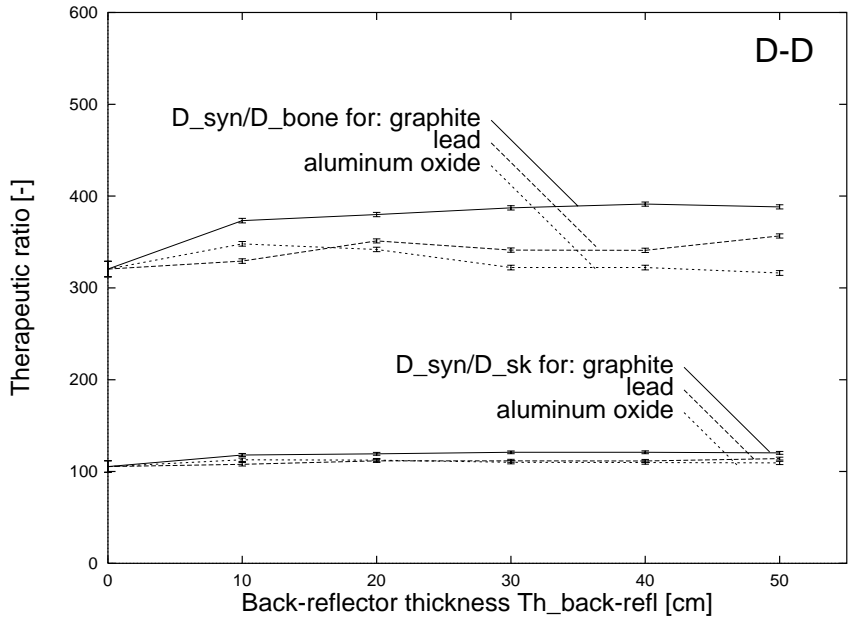

(a)

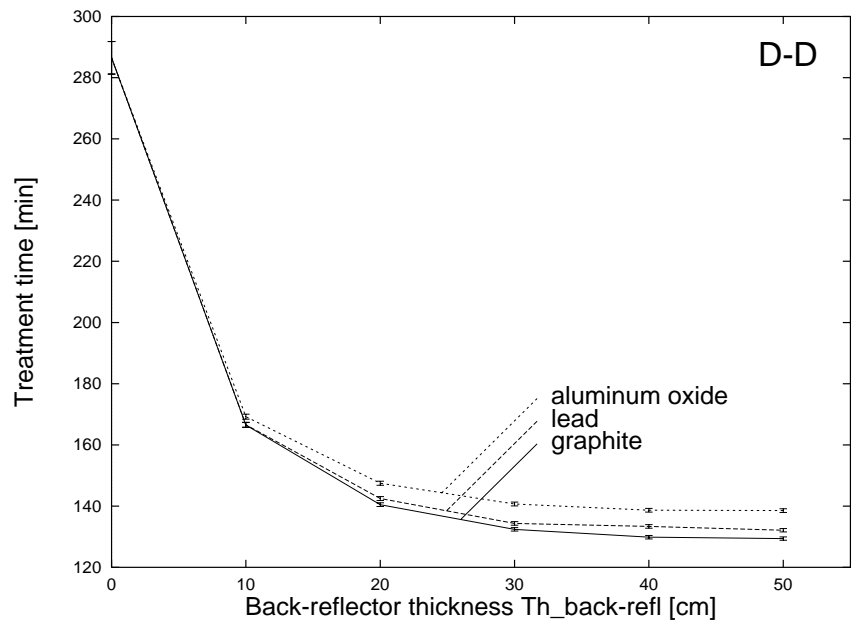

(b)

Figure 10: (a) Therapeutic ratios and (b) treatment time as a function of the back-reflector thickness and material. 
J.M. Verbeke - Optimization of beam-shaping assemblies for BNCS using the high-energy neutron sources D-D and D-T

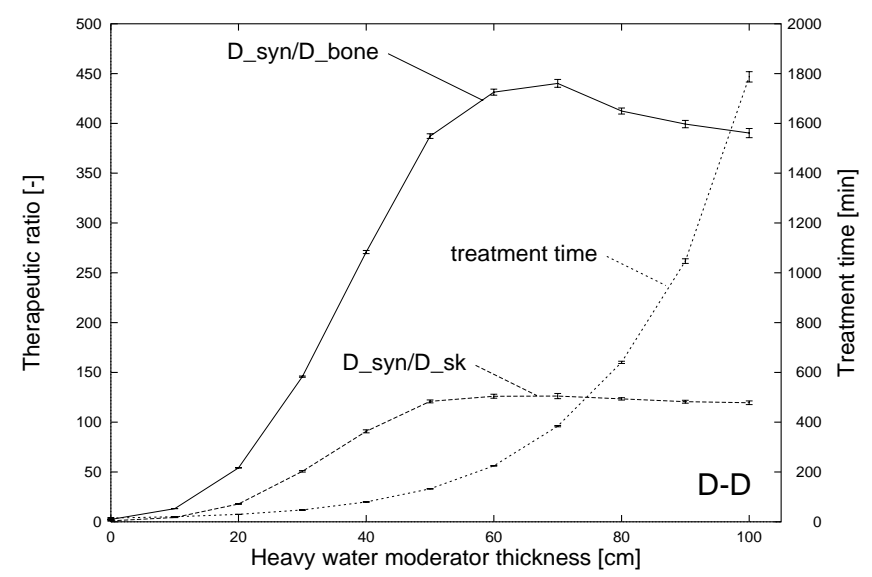

Figure 11: Therapeutic ratios and treatment time versus heavy water moderator thickness. 
J.M. Verbeke - Optimization of beam-shaping assemblies for BNCS using the high-energy neutron sources D-D and D-T

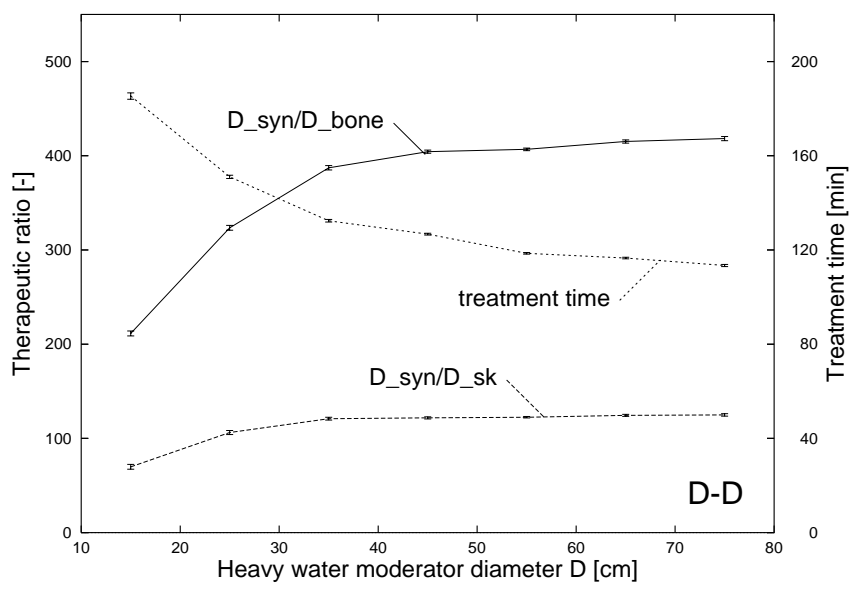

Figure 12: Therapeutic ratios and treatment time versus heavy water moderator diameter. 
J.M. Verbeke - Optimization of beam-shaping assemblies for BNCS using the high-energy neutron sources D-D and D-T

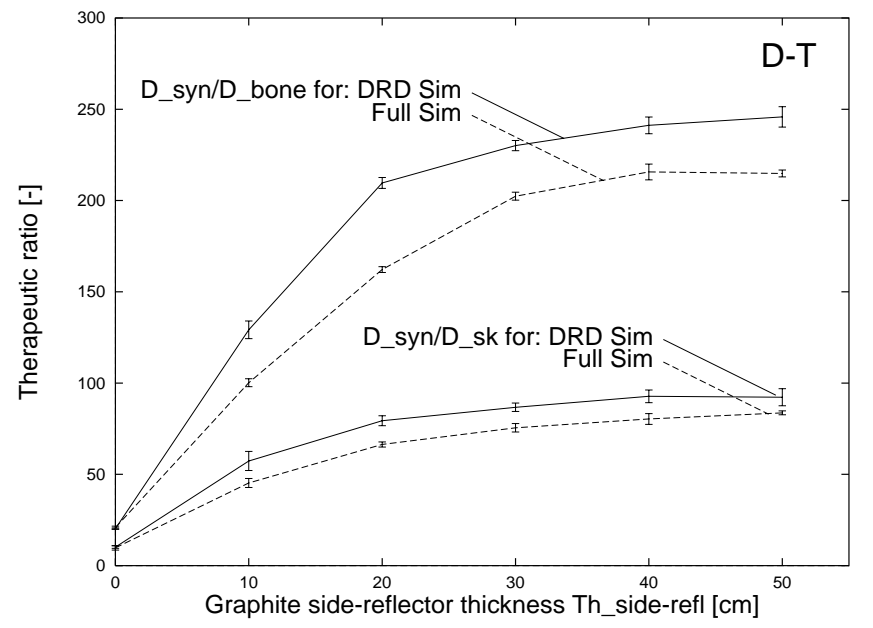

(a)

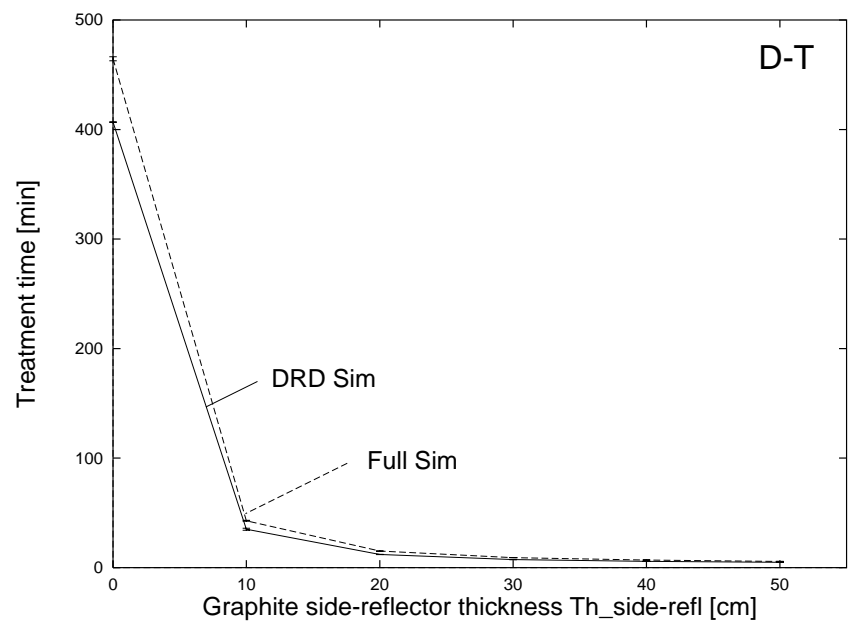

(b)

Figure 13: Comparison of (a) therapeutic ratios and (b) treatment times obtained with the dose-response database simulation and the full simulation methods using a lead front-reflector and a lead collimator. 
J.M. Verbeke - Optimization of beam-shaping assemblies for BNCS using the high-energy neutron sources D-D and D-T

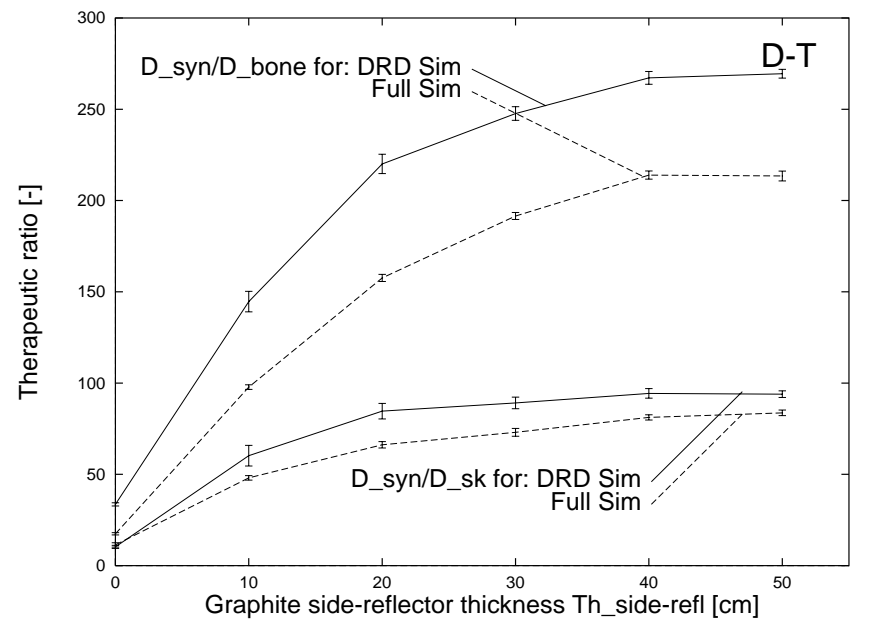

(a)

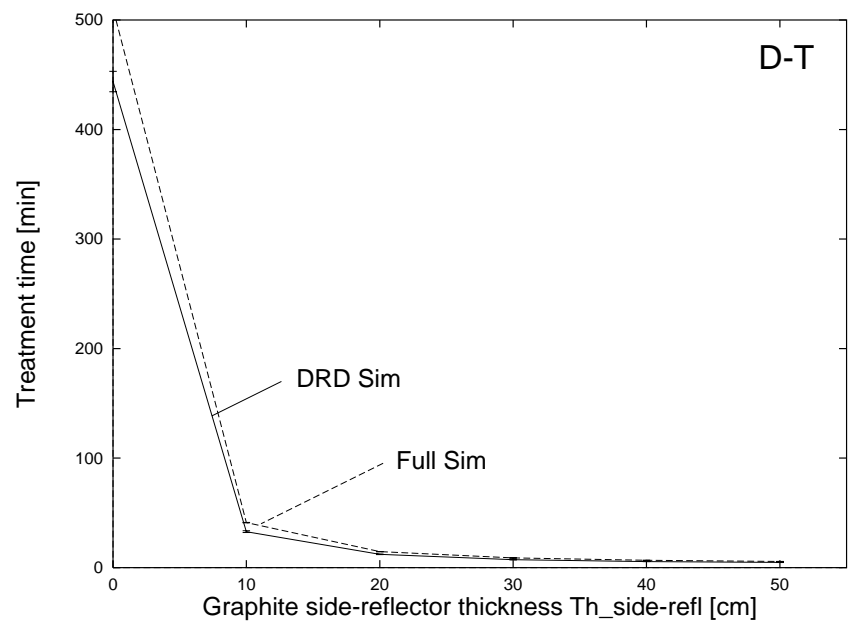

(b)

Figure 14: Comparison of (a) therapeutic ratios and (b) treatment times obtained with the doseresponse database simulation and the full simulation methods using a graphite front-reflector and a graphite collimator. 
J.M. Verbeke - Optimization of beam-shaping assemblies for BNCS using the high-energy neutron sources D-D and D-T

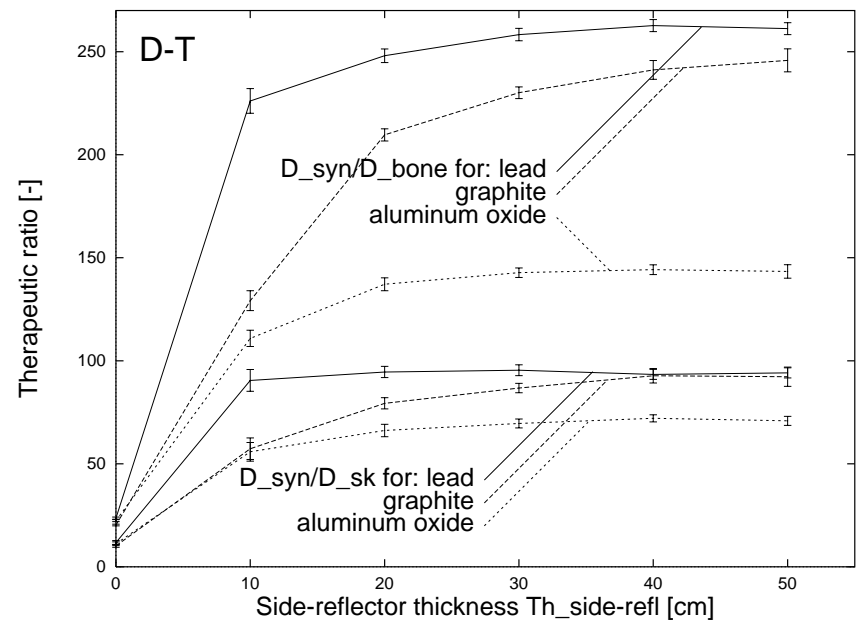

(a)

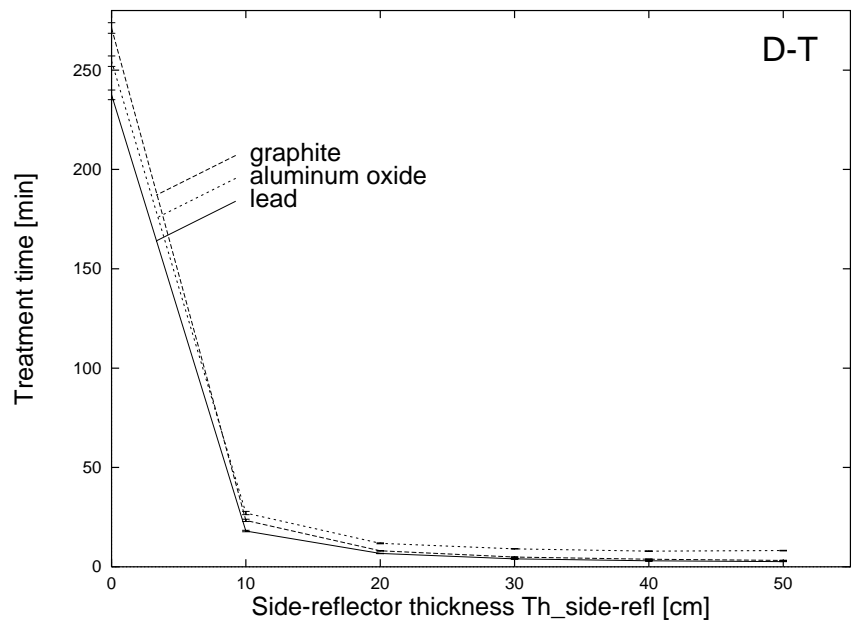

(b)

Figure 15: (a) Therapeutic ratios and (b) treatment time as a function of the side-reflector thickness and material. 
J.M. Verbeke - Optimization of beam-shaping assemblies for BNCS using the high-energy neutron sources D-D and D-T

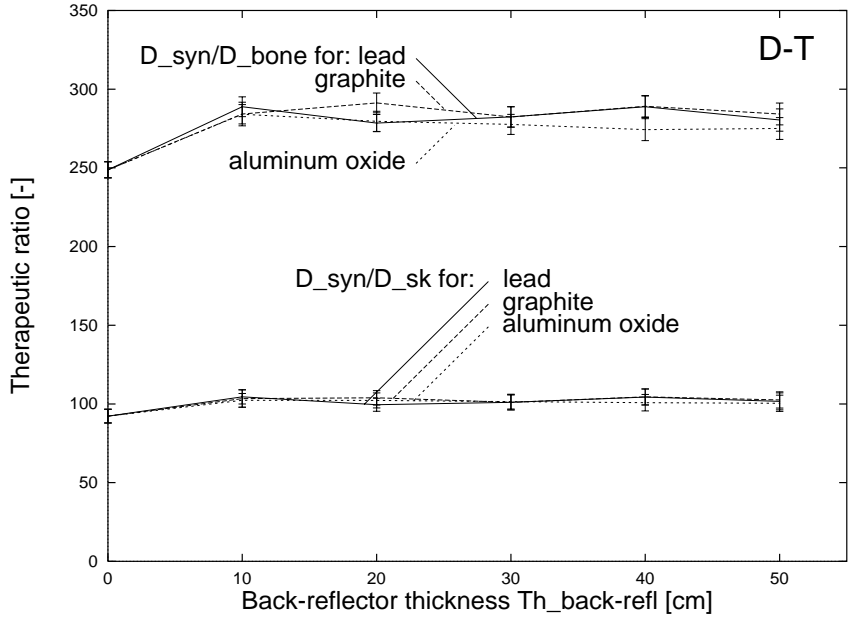

(a)

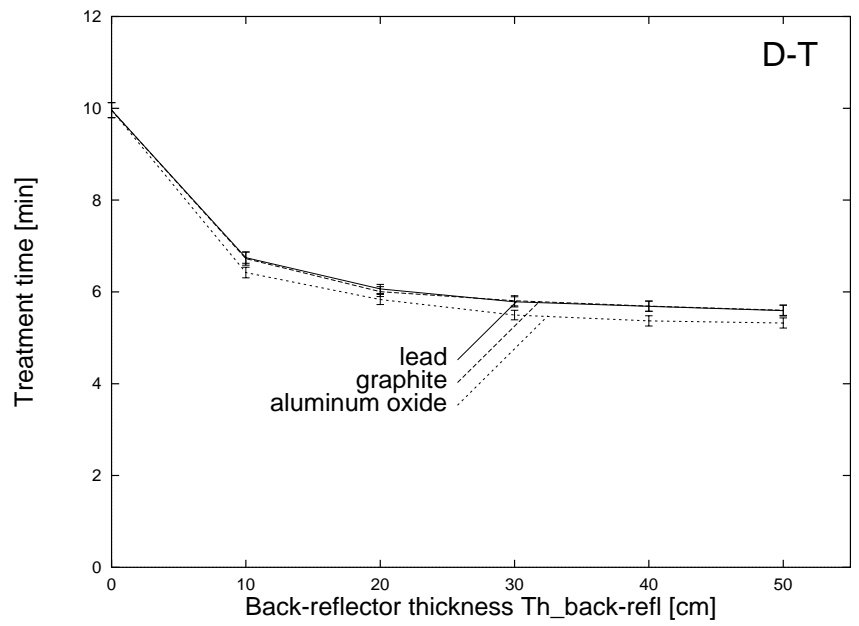

(b)

Figure 16: (a) Therapeutic ratios and (b) treatment time as a function of the back-reflector thickness and material. 
J.M. Verbeke - Optimization of beam-shaping assemblies for BNCS using the high-energy neutron sources D-D and D-T

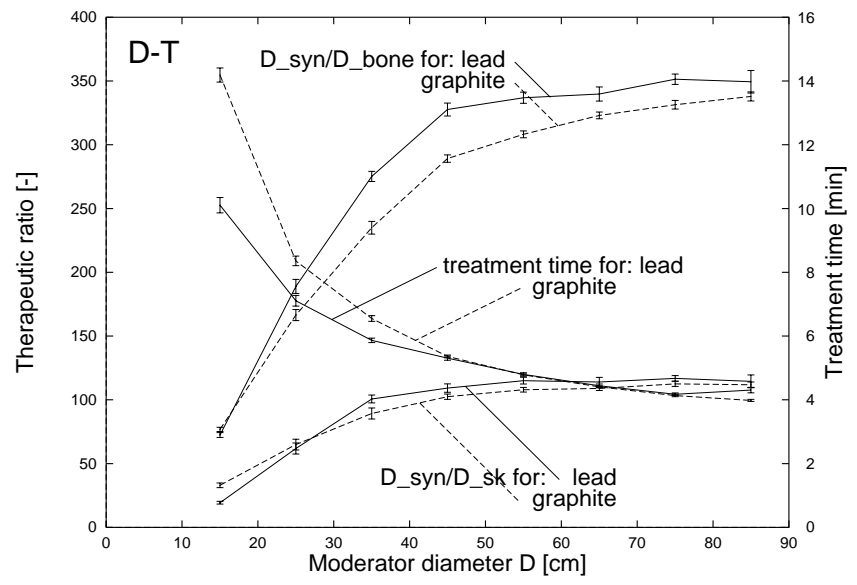

Figure 17: Therapeutic ratios and treatment time as a function of the moderator diameter and side-reflector material. A side-reflector thickness $\mathrm{Th}_{\text {side-refl }}$ of $30 \mathrm{~cm}$ is used for all simulations. 
J.M. Verbeke - Optimization of beam-shaping assemblies for BNCS using the high-energy neutron sources D-D and D-T

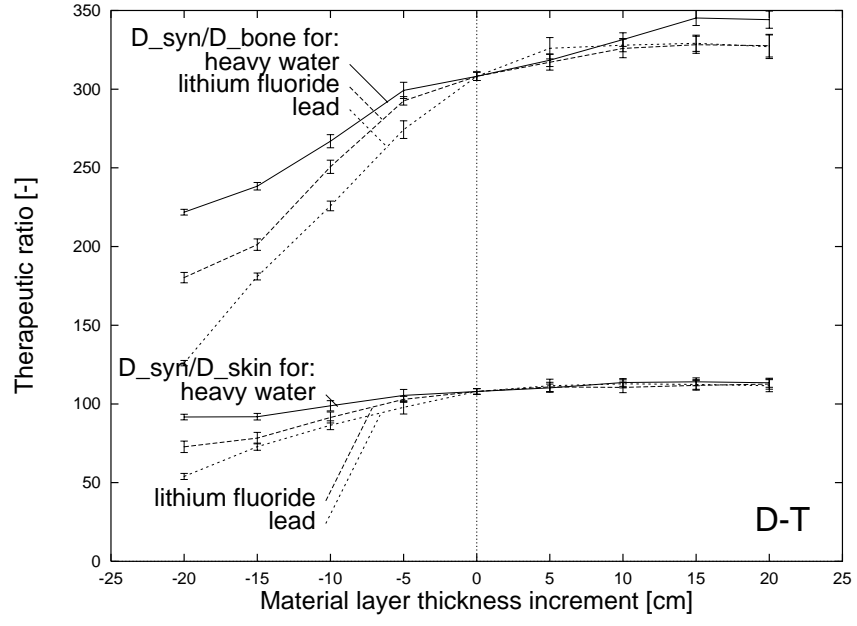

(a)

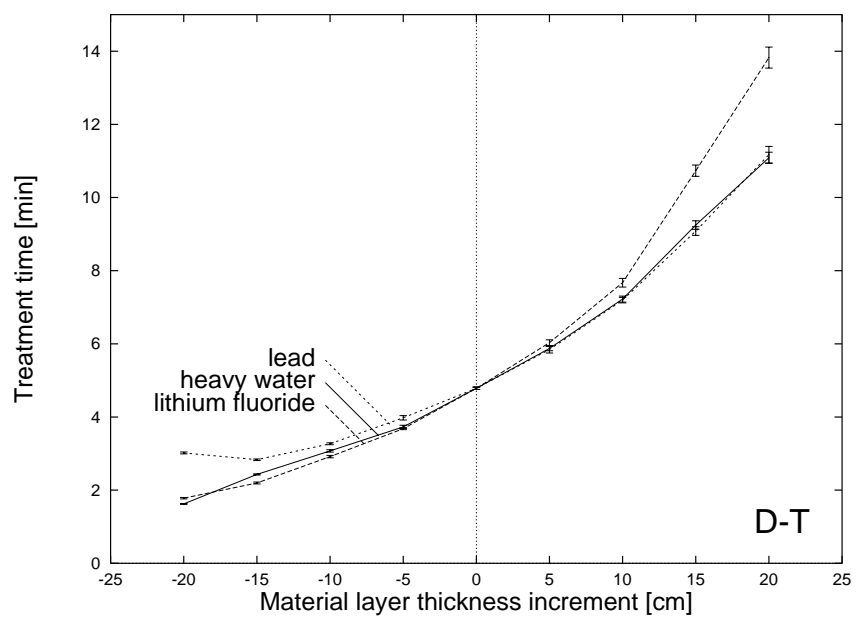

(b)

Figure 18: (a) Therapeutic ratios and (b) treatment time as a function of moderator material thickness increment. The reference moderator is composed of a 20-cm-thick layer of lead, followed by a 25 -cm-thick layer of lithium fluoride ${ }^{7} \mathrm{LiF}$, a 45 -cm-thick layer of heavy water and a 1-mm-thick layer of lead. 


\section{References}

[1] M. Davis, M. Chinol, "Radiopharmaceuticals for Radiation Synovectomy: Evaluation of Two Yttrium-90 Particulate Agents," J. Nucl. Med., 30, 1047-1055 (1989).

[2] J. Noble, A.G. Jones, M.A. Davis, C.B. Sledge, R.I. Kramer, E. Livni, "Leakage of Radioactive Particle Systems from a Synovial Joint Studied with a Gamma Camera," J. Bone Joint Surg. (Am), 65-A, 381-389 (1983).

[3] J.C. Yanch, S. Shortkroff, R.E. Shefer, S. Johnson, E. Binello, D. Gierga, A.G. Jones, G. Young, C. Viveiros, A. Davidson, and C. Sledge, "Boron Neutron Capture Synovectomy: Treatment of Rheumatoid Arthritis Based on the ${ }^{10} \mathrm{~B}(n, \alpha){ }^{7} \mathrm{Li}$ Nuclear Reaction," Med. Phys., 26, 364-375 (1999).

[4] Harris, D. Edward, Rheumatoid Arthritis, Saunders, Philadelphia (1997).

[5] J.C. Yanch, R.E. Shefer, and E. Binello, "Design of Low-Energy Neutron Beams for Boron Neutron Capture Synovectomy," Proc. SPIE - Int. Soc. for Opt. Eng., vol. 2867:3140 (1997).

[6] E. Binello, S. Shortkroff, A. Jones, C. Viveiros, A. Ly, C.B. Sledge, A. Davison, R.E. Shefer, and J.C. Yanch, "Research in Boron Neutron Capture Synovectomy," Proc. SPIE - Int. Soc. for Opt. Eng., vol. 2867:68-71 (1997).

[7] A.H.W. Nias, An Introduction to Radiobiology, John Wiley \& Sons, New York (1990).

[8] J.M. Verbeke, A. Chen, J. Vujic, and K.N. Leung, "Prediction of In-Phantom Neutron Beam Characteristics using In-Air Figure-Of-Merit for BNCS," Nucl. Tech., 131 (2), 269$276(2000)$.

[9] J.F. Briesmeister, "MCNP - A General Monte Carlo N-Particle Transport Code, Version 4B," LA-12625-M, Los Alamos National Laboratory (1997). 
[10] "Photon, Electron, Proton and Neutron Interaction Data for Body Tissues," ICRU 46, International Commission on Radiation Units and Measurements (1992).

[11] R.S. Caswell, J.J. Coyne, and M.L. Randolph, "Kerma Factors of Elements and Compounds for Neutron Energies Below $30 \mathrm{MeV}$," Int. J. Appl. Radiat. Isot., 33, 1227-1262 (1982).

[12] J.M. Verbeke, J. Vujic, and K.N. Leung, "Neutron Beam Optimization for BNCT using the D-D and D-T High Energy Neutron Sources," Nucl. Tech., 129 (2), 257-278 (2000).

[13] J.B. Marion, and J.L. Fowler, Fast Neutron Physics, Part I, Monographs and Texts in

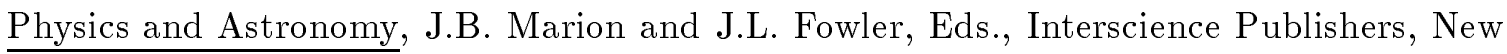
York (1960).

[14] H.B. Liu, D.D. Greenberg, J. Copala, and F.J. Wheeler, "An Improved Neutron Collimator for Brain Tumor Irradiations in Clinical Boron Neutron Capture Therapy," Med. Phys., 23, 2051-2060 (1996).

[15] J.M. Verbeke, K.N. Leung, and J. Vujic, "Development of a sealed-accelerator-tube neutron generator," Applied Radiation and Isotopes, 53 , 801-809 (2000).

[16] I. Auterinen, and P. Hiismaki, "Design of an Epithermal Neutron Beam for the TRIGA Reactor in Otaniemi," Proc. CLINCT BNCT Workshop, Helsinki, Finland, 1993, TKK-FA718, p. 14-24 (1994). 